\title{
Feasibility, acceptability, effect, and cost of integrating counseling and testing for HIV within family planning services in Kenya
}

Wilson Liambila

Population Council

Charlotte E. Warren

Population Council

Saiqa Mullick

Population Council

lan Askew

Population Council

Rick Homan

See next page for additional authors

Follow this and additional works at: https://knowledgecommons.popcouncil.org/departments_sbsr-rh

Part of the Demography, Population, and Ecology Commons, International Public Health Commons, Maternal and Child Health Commons, and the Women's Health Commons

How does access to this work benefit you? Let us know!

\section{Recommended Citation}

Liambila, Wilson, Charlotte E. Warren, Saiqa Mullick, lan Askew, Rick Homan, Ibrahim Mohammed, Robert Ayisi, Margaret Gitau, Josephine Kibaru, Mary W. Gathitu, Judith Maua, Helton Jilo, Juma Mwangi, John Njoroge, Anthony K. Wanyoro, Peter Mohammed Njuguna, and Andrew Mboche. 2008. "Feasibility, acceptability, effect, and cost of integrating counseling and testing for HIV within family planning services in Kenya," FRONTIERS Final Report. Washington, DC: Population Council. 


\section{Authors}

Wilson Liambila, Charlotte E. Warren, Saiqa Mullick, lan Askew, Rick Homan, Ibrahim Mohammed, Robert Ayisi, Margaret Gitau, Josephine Kibaru, Mary W. Gathitu, Judith Maua, Helton Jilo, Juma Mwangi, John Njoroge, Anthony K. Wanyoro, Peter Mohammed Njuguna, and Andrew Mboche 


\section{Feasibility, Acceptability, Effect and Cost of Integrating Counseling and Testing for HIV within Family Planning Services in Kenya}

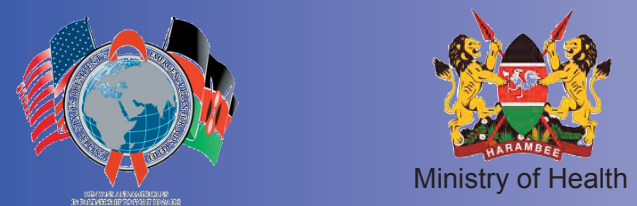
(1) Population Council FRONTIERS


This study was made possible by the generous support of the American people through the President's Emergency Plan for AIDS Relief (PEPFAR) and the United States Agency for International Development's U.S. Agency for International. Development (USAID) under the terms of Cooperative Agreement No. HRN-A00- 98-00012-00 and In-house Project No. 8816.53101. The contents are the responsibility of the FRONTIERS Program and do not necessarily reflect the views of USAID or the United States Government.

\section{Suggested citation:}

Liambila W., Askew I., Ayisi R., Gathitu M., Mwangi J., Homan R., et al. 2008: Feasibility, Acceptability, Effect and Cost of Integrating Counseling and Testing for HIV within Family Planning Services in Kenya. FRONTIERS Final Report, Washington DC, Population Council.

(C) 2008, The Population Council 


\section{Feasibility, Acceptability, Effect and Cost of Integrating Counseling and Testing for HIV within Family Planning Services in Kenya}

FRONTIERS/Population Council

Wilson Liambila

Charlotte Warren

Saiqa Mullick

Ian Askew

FRONTIERS/Family Health International

Rick Homan

\section{NASCOP/NLTP}

Ibrahim Mohammed

Robert Ayisi

Margaret Gitau
Division of Reproductive Health

Josephine Kibaru

Mary Gathitu

Judith Maua

Helton Jilo

Provincial and District Teams

Juma Mwangi

John Njoroge

Anthony K. Wanyoro

Peter Mohammed Njuguna

Andrew Mboche

July 2008 


\section{ACKNOWLEDGMENTS}

The activities reported in this document were accomplished following important inputs from various institutions and individuals. We wish to acknowledge the following officials who have played critical roles in the process of implementing the integration of counseling and testing for HIV within family planning services:

Ruth Wayua Muia and Roselyn Koech (Division of Reproductive Health); members of the Provincial Health Management Team (Central Province) especially the Provincial Medical Officer, Provincial Nursing Officer, and the Provincial Medical Records and Information Officer. We would specifically wish to thank Dr. Wilson Muriithi (Provincial AIDS/STDs Coordinator (PASCO) Central), and Florence Kungania (Trainer) for coordinating both training activities and the service delivery phase of the project.

Members of the District Health Management Team in Nyeri: the District Medical Officer of Health, District Public Health Nurse, District Medical Laboratory Technologist, Caroline Wanjohi (DASCO), Eunice Wachira (Nursing Officer/Trainer), and Agnes Gichogo (Nursing Officer/Trainer) are acknowledged for having taken part and supported project activities in all phases.

Members of the District Health Management Team in Thika: the District Medical Officer of Health, Damaris Kasyoka (DASCO/Trainer), Emma Waweru (District Public Health Nurse/Trainer), Elizabeth Kariuki (Deputy DDPHN/RHTS Coordinator), Esther Kabati (Trainer), Caroline Mbogori (Trainer) and Carolyne Macharia (in DPHN's office) are acknowledged for ensuring that all the activities that were planned were implemented on schedule and for supporting project activities in all the stages.

Members of the Provincial General Hospital Team: Lucy Njue (Nursing Officer/RH Trainer) and Jennifer Kiruri (Nursing Officer/Counselor/Trainer) are acknowledged for having taken part in all the training activities. The Medical Superintendent and the Nursing Officer in-charge of the PGH Nyeri are thanked for supporting project activities and specifically for allowing the training team to use the hospital for practical sessions.

We wish to thank Wasunna Owino (Constella Futures) and Nzoya Munguti (MOH) for reviewing the cost analysis section and providing valuable suggestions, and Amber Peterman and Erick Oweya (Population Council) for undertaking many analyses of the data.

Grace Kihindas (RH Trainer), Judith Muganda, Janet Munyasya (Administrative Assistant Population Council), and Hannah W. Kihia (DASCO Office, Thika) are thanked for having played various roles in the project activities.

USAID and PEPFAR are acknowledged for having provided financial support for the project activities.

Finally, we would like to thank all FP clients and health providers, as well as the research assistants, for agreeing to participate in and implementing the study. 


\section{SUMMARY}

Integrating counseling and testing (CT) for HIV into family planning (FP) services potentially increases the range of services available for FP clients, many of whom are at risk of STIs including HIV in high prevalence settings. Systematic evidence about offering CT in FP settings has remained extremely limited, despite the widespread interest in this model of FP-HIV integration. FRONTIERS supported the Division of Reproductive Health (DRH) and the National AIDS and STI Control Program (NASCOP) of the Kenya Ministry of Health (MOH) to design, implement and compare two models of integrating CT for HIV within FP services in 23 health facilities in Nyeri and Thika Districts of Central Province, Kenya in terms of their feasibility, acceptability, cost and effect on the voluntary use of CT, as well as the quality of FP services. The study utilized a pre-post intervention design to obtain information from FP providers and their clients in 2006 to 2007.Data were collected through provider-client observations (554 at baseline and 530 at endline) and client exit interviews (552 at baseline and 530 at end line), pre and post intervention interviews and focus group discussions with health providers, and a health facility assessment of the readiness of facilities to offer HIV CT within FP services.

Introduction and implementation involved: (a) holding sensitization meetings at national, provincial and district levels; (b) reviewing and developing training materials; (c) application of the Balanced Counseling Strategy (BCS) Plus approach; (d) modification of facility registers to record the required data; and (e) training of health providers. The $\mathrm{MOH}$ provided all required equipment and supplies, including HIV rapid test kits and FP commodities.

Two models were pilot-tested. The "testing" model was implemented in Nyeri District, an area with relatively few VCT sites. In this model, FP clients were educated about HIV prevention generally, and CT in particular, and offered HIV CT during this consultation by the FP provider. The "referral" model was implemented in Thika district, an area with good accessibility to VCT services. In this model, FP clients were educated about HIV CT, and those interested were instead referred to a specialized CT service, either within the same facility or to another CT service (at another health facility or a stand-alone VCT center).

The study demonstrated that both models were feasible and acceptable to providers and to clients as means of integrating and linking HIV prevention counseling, condom promotion and counseling and testing with FP services, and are effective in increasing quality of care and service utilization. Specific findings showed that:

Integrating counseling and testing for HIV into FP services is feasible and acceptable: The majority of facilities had the capacity to integrate HIV prevention counseling and provision of or referral for CT services within existing FP services. Provision of both services jointly was perceived by clients to be beneficial and attractive and was welcomed by providers as an opportunity to provide a comprehensive service that better meets their clients' needs. Existing differences between clinics in each district meant that some were better prepared than others to offer integrated services. 
Quality of family planning counseling improved: Significant improvements were observed in the quality of FP counseling and in the rapport established with clients in both models. Discussion of reproductive intentions remained a weakness however.

Quality of counseling on STI/HIV issues improved: Levels of counseling on STI/HIV issues were low at baseline. After introduction of the BCS Plus approach, there were marked increases in discussions about STIs and HIV/AIDS generally and about the risk factors, but discussions of the client's personal sexual history and behaviors remained low.

Counseling on condoms and dual protection and their use improved: Condoms were rarely mentioned or discussed at the baseline, especially in Thika district. Training and use of the BCS Plus significantly increased discussions around condoms and their use. Reported use of condoms at last sex, in last month, and with another contraceptive method all increased significantly.

Counseling on HIV CT increased during FP consultations: One of the focus items of the intervention was to increase discussions about HIV counseling and testing during the FP consultations. At baseline, the proportion of consultations in which HIV CT was mentioned was already quite high, at 39 percent overall. Introduction of the intervention led to substantial increases, with HIV CT being mentioned in 88 percent of all consultations at endline. Discussions of the client's serostatus increased from 24 to 81 percent of consultations.

Use of the BCS Plus approach significantly improved the quality of integrated services: Summary scores were calculated for 27 items that providers were trained to cover when providing integrated services. The mean summary scores for those consultations when the BCS Plus approach was used were much higher (18.86) than for consultations when it was not used (12.19), clearly indicating that the BCS Plus approach is an effective means for improving the quality of counseling when providing integrated services. The approach was particularly effective in Thika district, where quality of care scores almost doubled when it was used.

Clients' offered and obtaining HIV CT increased: Approximately two-thirds of all new clients had ever had an HIV test, and this did not change over time, nor was there any difference between the districts, suggesting that there were no external influences during the study period. Most repeat clients had been exposed to the interventions and at endline there were significant increases in the proportions reporting ever been tested after the intervention (from $43 \%$ to 67\%), and especially among those exposed to the testing model. There were substantial and significant increases in the proportions of clients offered HIV testing during the consultation, from one percent at baseline to 39 percent (repeat) and 48 percent (new) at endline. Providers implementing the testing model were more likely than those implementing the referral model to offer HIV testing, perhaps because they were able to offer the test themselves. For both models, the proportions of new clients being offered HIV testing $(34 \%-74 \%)$ were higher than for repeat clients (27\% - 56\%), which may be because the majority of repeat clients use injectables and pills, and so the provider may have assumed that the client had been offered testing previously. Overall, 50 - 72 percent of FP clients who were offered HIV testing accepted to have a test. Repeat clients and those attending referral model clinics were more likely to accept a test, which may suggest a preference for being tested anonymously at another site. The proportions of all FP clients actually having an HIV test after the FP consultation were 20 percent in the referral model and 35 percent in the testing model, with little difference between new or repeat clients. Overall, approximately one-third of those choosing to have an HIV test had never had a test 
before; this proportion increased to one half for those attending referral model clinics. These data clearly indicate that both models are effective in substantially increasing the number of women accessing HIV counseling and testing, including many women who had never before had a test, through offering the service during FP consultations.

Incremental costs for integrating CT into FP services are affordable: On average, the additional time required during an FP consultation to provide the CT service was reasonable 3.03 minutes for the testing model (from 12.4 to 15.4 minutes) and 4.18 minutes in the referral model (from 13.2 to 17.4 minutes), and the additional costs per FP client for this time were affordable, at $\$ 0.41$ for the testing model and $\$ 0.24$ for the referral model. For planning scalingup of the testing model, the estimated incremental cost per FP client who is also counseled and tested for HIV ranges from $\$ 5.60$ (hospital) to $\$ 9.53$ (dispensary). This compares favorably with an estimated cost per client at stand-alone VCT sites of \$27.

Drawing from the lessons learned, the key programmatic recommendations for institutionalizing and scaling up this approach are:

- Ensuring that national, provincial and district level managers and trainers from both DRH and NASCOP lead activities builds capacity for introducing new service delivery protocols.

- District and facility action plans can ensure resource mobilization and secure commitment to providing integrated services.

- The Balanced Counseling Strategy Plus approach and tools has enhanced the range of services offered to FP clients and significantly improved the quality of care.

- On-the-job updates by MOH trainers rapidly expanded the number of health providers able to provide HIV CT for FP clients, but for sustainability the existing FP service delivery protocols need to be reviewed and revised to integrate the HIV service procedures.

- Continuous availability of both FP and HIV CT commodities ensured an attractive and accessible service.

- Both models are effective in increasing the number of women testing for HIV, both for the first time and for a repeat test.

- Clinics that can offer HIV testing on-site should also consider offering clients the option of being referred elsewhere for the test, given the evidence suggesting that anonymity is important for these FP clients who may meet the same provider on several occasions.

The preliminary findings and lessons learned were communicated to key stakeholders in August 2007. Drawing on this evidence, the DRH and NASCOP recommended that integration of HIV CT within FP services should be adopted, especially using the testing model where possible, and scaled up nationwide. A meeting in September 2007 planned for national and provincial-level training of trainers (TOTs). By end of October 2007, about 40 provincial-level TOTs from the remaining seven provinces had been trained by NASCOP and DRH. By mid-July 2008, the USAID-funded APHIA II partners had supported the Provincial Health Management Teams to train 93 providers in Central province and 33 providers in Nairobi province. By the end of 2008, Nairobi province plans to train a further 90 providers, Eastern Province will train a further 20 TOTs and 200 providers, and Coast Province will train 120 providers. Lessons from this study have been presented at several national and international workshops and conferences. 


\section{CONTENTS}

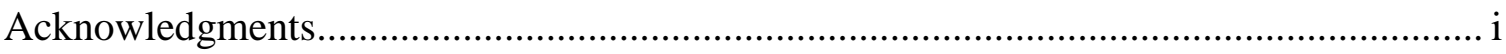

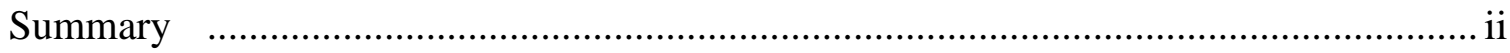

Acronyms and Abbreviations ............................................................................... vii

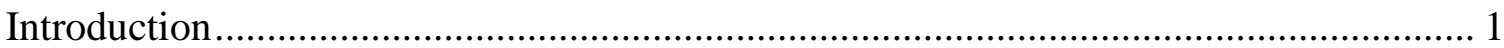

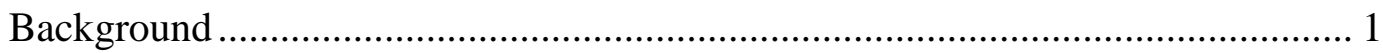

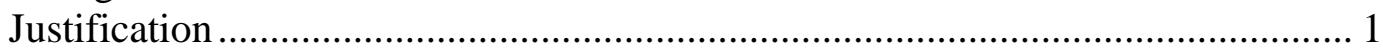

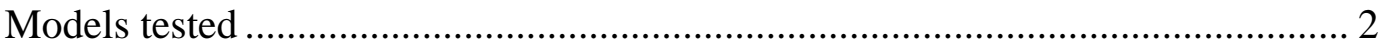

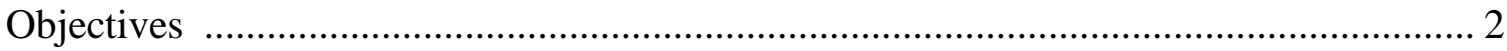

Development and implementation of the two models ............................................... 3

Sensitization of the National, Provincial and District teams ................................... 3

Models to be compared .................................................................................. 3

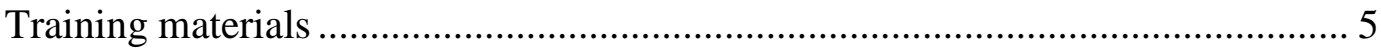

Balanced Counseling Strategy Plus (BCS-Plus) ………….................................. 5

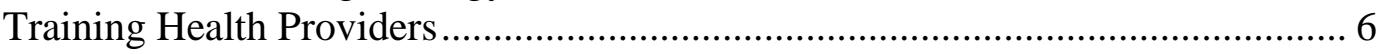

Strengthening basic supplies and commodities ....................................................... 7

Strengthening routine data collection on FP and VCT services ............................ 7

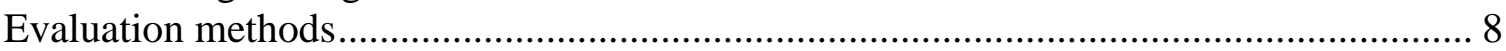

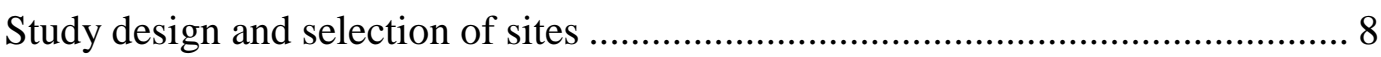

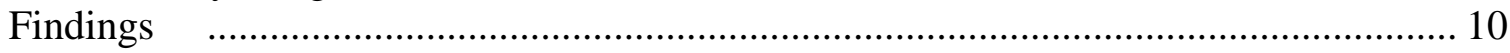

Readiness to offer integrated services.............................................................. 10

Acceptability of services to clients ................................................................... 11

Acceptability to providers............................................................................... 12

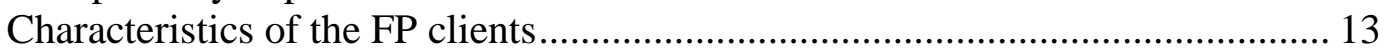

Quality of FP Counseling............................................................................. 14

Quality of STI/HIV Counseling ........................................................................ 15

Quality of counseling about condom use .......................................................... 16

Quality of counselling on HIV C\&T ............................................................... 17

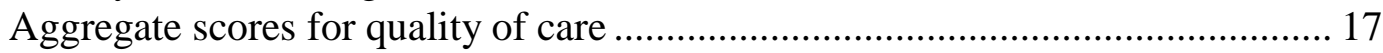

Cost analysis and incremental costs for scaling up............................................. 21

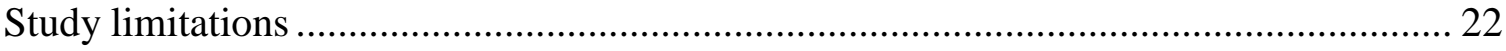

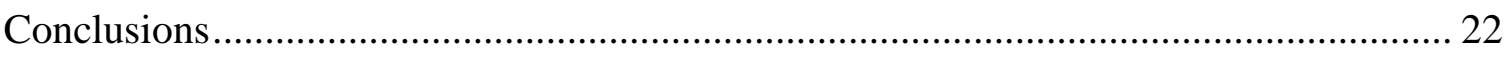

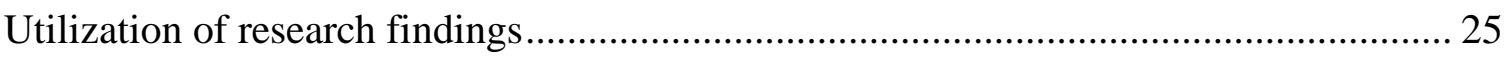

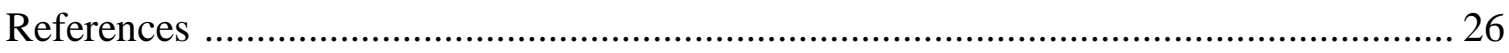

Appendix 1: Algorithm for the Balanced Counseling Strategy Plus ..................... 27

Appendix 2: Example of BCS-plus counseling cards......................................... 28

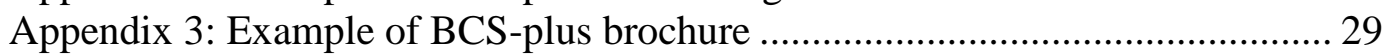

Appendix 4: Study facilities and client load ....................................................... 30

Appendix 5: Availability of equipment, supplies and FP and STI/HIV services . 31

Appendix 6: Measuring and analyzing incremental costs ..................................... 34 


\section{LIST OF TABLES}

Table 1: Pre and post test mean scores for participants attending training sessions by district 6

Table 2: Sample sizes for client-provider observations and client exit interviews. . .9

Table 3: Availability of basic equipment, supplies, infrastructure and services for FP and HIV/STIs.

Table 4: Characteristics of clients by model

Table 5: Proportions of consultations in which FP issues were discussed .14

Table 6: Proportion of consultations in which client rapport established 15

Table 7: Discussion of STI/HIV issues with FP clients

Table 8: Counseling and provision of condoms .............................................................

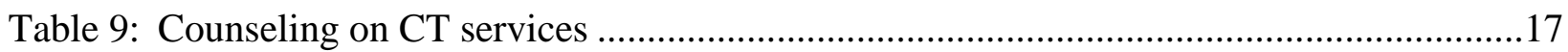

Table 10: Summary scores for quality of care components before and after intervention ............18

Table 11: Clients' use of condoms (percentages) .....................................................................19

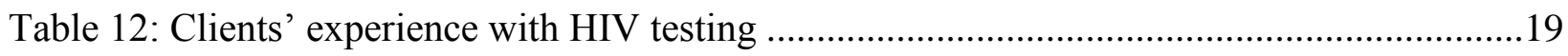

Table 13: Incremental cost per facility of scaling-up by model and level of care......................21 


\section{ACRONYMS AND ABBREVIATIONS}

$\begin{array}{ll}\text { AIDS } & \text { Acquired Immune Deficiency Syndrome } \\ \text { ARV } & \text { Antiretrovirals } \\ \text { BCS } & \text { Balanced Counseling Strategy } \\ \text { CT (C\&T) } & \text { Counseling and Testing (for HIV) } \\ \text { DASCO } & \text { District AIDS Control Program } \\ \text { DHMT } & \text { District Health Management Team } \\ \text { DP } & \text { Dual Protection } \\ \text { DRH } & \text { Division of Reproductive Health } \\ \text { FGDs } & \text { Focus Group Discussions } \\ \text { FP } & \text { Family Planning } \\ \text { HIV } & \text { Human Immunodeficiency Virus } \\ \text { IUD } & \text { Intra-Uterine Contraceptive Device } \\ \text { KDHS } & \text { Kenya Demographic and Health Survey } \\ \text { KEMSA } & \text { Kenya Medical Supplies Agency } \\ \text { MOH } & \text { Ministry of Health } \\ \text { NASCOP } & \text { National AIDS Control Program } \\ \text { OPD } & \text { Outpatient Department } \\ \text { PASCO } & \text { Provincial AIDS Control Program } \\ \text { PEPFAR } & \text { President's Emergency Plan for AIDS Relief } \\ \text { PGH } & \text { Provincial General Hospital } \\ \text { PHC } & \text { Primary Health Care } \\ \text { PHMT } & \text { Provincial Health Management Team } \\ \text { PMTCT } & \text { Prevention of Mother to Child Transmission (of HIV) } \\ \text { RH } & \text { Reproductive Health } \\ \text { RHTS } & \text { Reproductive Health Training and Supervision } \\ \text { RTI } & \text { Reproductive Tract Infections } \\ \text { VHO } & \text { Sexually Transmitted Infections } \\ \text { RH Counseling and Testing (for HIV) }\end{array}$




\section{INTRODUCTION}

\section{Background}

In Kenya, HIV prevalence rates are high in the general population (estimated at 6.7 percent in 2003) but uptake of voluntary counseling and testing for HIV (VCT) remains low at 13 percent (KDHS, 2003). The 2003 Kenyan DHS found that women were less likely to have heard of VCT (48 percent) and less likely to have used a condom in their last high risk sex encounter (24 percent) than men. Condom use as a contraceptive method was reported to be low at 10.5 percent (KDHS, 2003). Almost 40 percent of women in the reproductive age group are using family planning (FP) services, and most users (58 percent) obtain their method from public sector health facilities. The majority use the injectable or pill, methods that require frequent visits to clinics (KDHS, 2003) and do not protect against HIV and STI acquisition. Approximately 20 percent of births are unwanted and 25 percent are mistimed.

Despite this evidence, relatively little attention has been paid to the opportunities of offering HIV counseling and testing (CT) within the context of other reproductive health $(\mathrm{RH})$ services attended by people at risk of HIV. Thus, integrating counseling and testing for HIV within FP services potentially increases the range of services available for FP clients, many of whom are at risk of STIs including HIV. Evidence of the feasibility, acceptability, cost and effect of offering counseling and testing in FP settings has over the years remained limited.

At present, the policy environment is conducive to the integration of services and the relevant departments within the Kenyan Ministry of Health $(\mathrm{MOH})$ work together to seek common benefits. The National Reproductive Health Strategy (1997-2010) highlights integration of services and quality of care as key components of its strategy (MOH, 1996). The current National Health Sector Strategic Plan (NHSSP II) 2005-2010 also highlights integration of HIV/AIDS programs into maternal and child health as a key output to be achieved during the plan period (MOH, 2005). In addition, the $\mathrm{MOH}$ policy on reproductive health emphasizes the need for integrating RH and HIV/AIDS information and services. The Ministry of Health draft strategy document entitled "Strategy for the Integration of Voluntary Counseling and Testing Services and Family Planning Services" defines integration as "the incorporation of some or all of the different FP services into existing VCT services and vice versa (MOH, 2005)."

A study on integration of HIV and FP services in Ethiopia (Bradley et. al., 2006) categorized integration into three levels, facility integration (co-location of HIV and FP services within the same facility), room integration (offering HIV and FP services in the same room), and counselor integration (offering HIV and FP services by same counselor in one consultation). The study showed that, relative to co-locating services in the same facility, those integrating services at room and counselor-levels were $1.9-7.2$ times more likely to serve clients wanting HIV testing.

\section{Justification}

The MOH draft strategy on integration of VCT and FP services states that "A major concern of the Kenya government, program implementers, and donors is that the VCT program is evolving as a parallel program to other efforts, especially those of the $\mathrm{MOH}$ that are aimed at decentralizing and integrating services." To date, only models of FP integration into VCT 
services have been developed and tested in Kenya (Reynolds et al, 2006). Integration of CT into FP has not yet been addressed and so evaluation of alternative models for this approach will assist the MOH to make programmatic decisions on cost effective approaches to improving uptake of HIV CT and improving the quality of FP services. To review evidence and provide guidance on FP-HIV integration broadly, the MOH has established an RH-HIV sub-committee within the RH-HIV Working Group, chaired jointly by the National AIDS and STI Control Program (NASCOP) and the Department of Reproductive Health (DRH), and reporting to the Head of the Department of Preventive and Promotive Services. The main aim of the committee is to develop an integrated RH/HIV program that will ensure improved coordination and collaboration among key agencies and organizations offering RH and HIV information and services.

\section{Models tested}

Two models were developed and tested in response to the MOH's interest in being able to offer different models of integration of CT into FP, depending on the context in which CT is already available. Both models include common components of a strengthened FP consultation plus counseling about and an assessment of STI/HIV risk behaviors. The "referral" model then provides information about HIV CT to all FP clients, with referral to existing VCT services elsewhere for those interested in being tested. This model is most relevant for those facilities in areas that already have a number of VCT services accessible to the clients and that do not necessarily need to offer HIV CT on-site; this model was tested in Thika district. The "testing" model also provides information on HIV CT, but then offers on-site testing and post-test counseling by the FP provider. This model is most relevant for facilities located in areas that do not have many VCT sites available and was implemented in Nyeri District.

\section{OBJECTIVES}

The overall objective of the project was to assess the feasibility, acceptability, effect and cost of integrating VCT information and services into FP services. The specific objectives were:

1. To develop and implement a model of integration that educates FP clients about VCT and offers them counseling and testing for HIV within the routine visit by a FP provider.

2. To develop and implement a model of integration that educates FP clients about VCT and refers interested clients for testing and post-test counseling to a specialized VCT service.

3. To describe the feasibility of implementing each of the two models and provider perspectives on their implementation.

4. To assess the implementation of the two models in a number of health care delivery settings in terms of their acceptability to clients, effectiveness in increasing VCT uptake, and incremental costs.

5. To assess the effect of integrating VCT on the quality of FP services received.

6. To disseminate and utilize results to create the conditions for scale up. 


\section{DEVELOPMENT AND IMPLEMENTATION OF THE TWO MODELS}

\section{Sensitization of the National, Provincial and District teams}

Following discussions at the national level with the FP Working Group and the VCT Integration Sub-committee, a sensitization meeting on integrating VCT activities into FP services was held in Nyeri in November 2005. Key stakeholders included the Provincial Health Management Team (PHMT) of Central Province, the District Health Management Teams (DHMTs) from Thika and Nyeri Districts, NASCOP, DRH and FRONTIERS. The purpose of the meeting was to discuss the national VCT/FP integration strategy, the proposed models of integration, planning issues and data collection requirements. During the meeting, members of the provincial and district teams discussed: the current number of VCT sites, strengths and opportunities for the proposed project, challenges, solutions and their expected roles and responsibilities. An outcome of this meeting was the decision to proceed with developing and comparing two models.

\section{Models to be compared}

The conceptual framework on which the two models were based is described in Figure 1 below.

The Referral Model: This model was implemented in Thika district, where the majority of facilities already had on-site VCT services and there were a number of stand-alone VCT sites. Family planning providers were trained to provide standard HIV/STI prevention and VCT awareness information, followed by individual risk assessment and a strengthened focus on promoting dual protection. HIV/STI prevention information was also incorporated into group health talks that usually take place at all clinics. All clients expressing an interest in VCT were referred internally to the on-site VCT service or externally to VCT services at other facilities for pre-test counseling, testing and post-test counseling. Health providers had a list of clinics and centers providing VCT services within the district, which was shared with the clients.

The Testing model: The testing model was implemented in Nyeri district, where on-site VCT services were not readily available. Providers from health centers and dispensaries (all without on-site VCT facilities) and from the Provincial General Hospital in Nyeri (which had on-site VCT) were trained to provide standard HIV/STI prevention and VCT awareness information, individual risk assessment and dual protection during all FP consultations. Clients who expressed an interest in VCT during the consultation were offered pre-test counseling, testing, and post-test counseling by the FP provider herself, either on the same day or at a later date. Clients were asked whether they preferred their FP provider to test them or if they wanted to be referred to another VCT service. HIV-positive clients were referred for follow-up and treatment in appropriate health facilities that had comprehensive care centers (CCCs). 
Figure 1: Conceptual Framework for Integrating HIV CT into FP Services

- Improved health status of population

- Desired fertility intentions addressed

- Enhanced HIV and STI preventive efforts

- Promotion of safer sex and dual protection

- HIV status known

- Increased uptake of counseling and testing services for HIV

- Improved quality of family planning services

- Training of health Providers

- Availability of FP Commodities and rapid test kits for HIV and other supplies

- Use of BCS Job aids

- Training manuals and protocols

- Supervision and monitoring

- Modification of registers

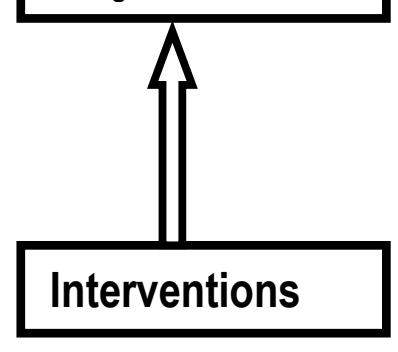

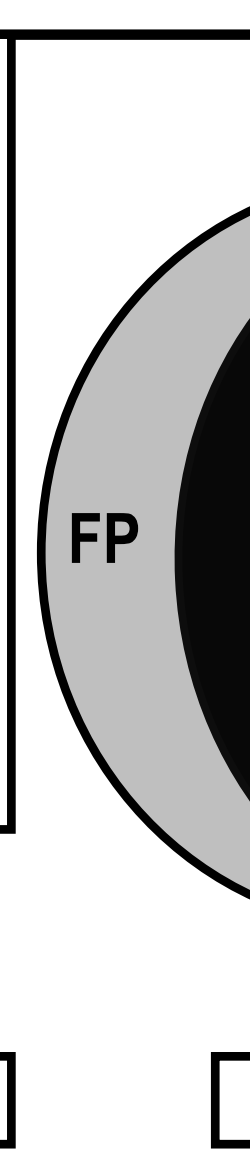

Routine Information \& education for HIV \& risk assessment/ verbal screening for RTIs/STIs

Routine offer of HIV testing \& counselling services in FP consultations

FP clients to benefit from IEC and services for HIV in one visit preferably by one provider

Referrals for services not offered on site

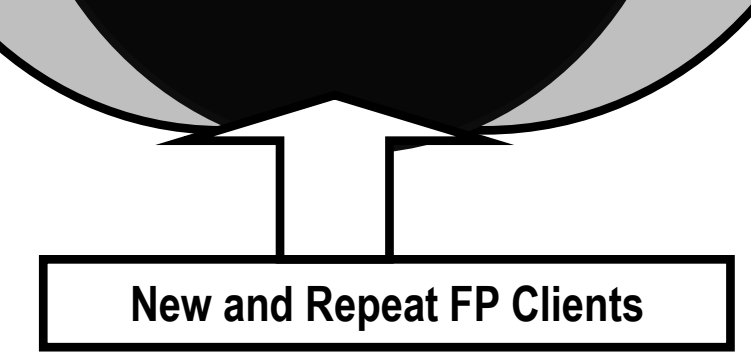

- Enabling health policies

- Functional management systems

- Functional procurement and logistics systems

- Changes in service delivery

- Availability of space and infrastructure

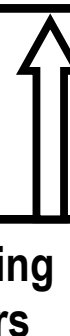

Key: FP= Family Planning Information and Services; HIVIAIDS= Information and Services. The two services overlap in the shaded area which represents additional services that an FP client receives. 


\section{Training materials}

National policies and guidelines relevant to HIV testing and family planning were reviewed in December 2005, including: FP Guidelines for Service Providers 2004; National Guidelines on Reproductive Tract Infection Services 2006; National PMTCT Training Manual 2005; and the National Trainers Manual on Integrating FP into VCT (draft 2004). Subsequently three sets of training materials were developed between January and April 2006: a trainers' manual; a trainees' manual; and a national protocol. All topics in the training manuals have been prepared as PowerPoint presentations to standardize the training package for future use during scale-up. The review and development of these training materials involved many institutions, including DRH, NASCOP, PHMT Central, DHMTs (Nyeri and Thika) and FHOK among others. Revision of the training materials also took into account findings from the needs assessment.

\section{Balanced Counseling Strategy Plus (BCS-Plus)}

The Balanced Counseling Strategy (BCS) is a counseling approach that uses a toolkit consisting of several job aids to facilitate structured FP consultations. The BCS was developed and validated in Latin America by the Population Council (León, 1999 and León, et al. 2003) ${ }^{1}$. This version of the BCS job aids was first adapted in South Africa for use in high HIV and STI prevalence settings, and then reviewed, modified and adapted by the trainers in Kenya. The modified version, referred to as BCS-Plus, seeks to improve the overall quality of FP services through increased choice and discussion of FP methods, strengthened integration of issues surrounding HIV and STIs into FP, increased discussion of dual protection, and improved prevention and control of RTIs/STIs among FP clients through early detection and management.

The modified BCS-Plus toolkit consists of a: (a) an algorithm to guide the provider through a semi-structured consultation; (b) a set of method-specific cards to facilitate counseling; and (c) brochures to be taken away by the client that describe the chosen method in detail.

Algorithm: The algorithm summarizes the key steps that a service provider should take to implement the BCS-plus during a counseling session. The steps are organized chronologically under three stages: Pre-choice; Method Choice; and Post-Choice. Each step describes the actions the provider needs to take or question s/he needs to ask. Depending on the client's response, the algorithm describes the following steps to take. The algorithm is described in Appendix 1.

Counseling cards: There are 14 counseling cards in the toolkit. The first card contains six questions that the service provider will ask to rule out if a client is pregnant. The 13 methodspecific cards are used to help narrow down choice of the appropriate method for the client. Each method card has an illustration of the method on the front side of the card. The backside of the card contains a description of several basic attributes or characteristics of the method. This allows the client to get an idea about the method. An example is given in Appendix 2.

Brochures: The toolkit includes 13 brochures, one for each FP method. Each brochure contains general information on the FP method, limitations on its use, how to use the method, side effects,

\footnotetext{
${ }^{1}$ See: http://www.popcouncil.org/frontiers/bestpractices/BCSpage_082007.html.
} 
other benefits, key points for follow-up, and warning signs for when to seek medical attention. An example of the FP method brochure for the IUD is given in Appendix 3.

\section{Training Health Providers}

A total of 75 health care providers, 47 in Thika and 28 in Nyeri, were trained. Implementation of the two models started in mid-May 2006 after the first group of trainees had qualified. Training focused on service integration, updates in FP method effectiveness and WHO eligibility criteria, RTIs and HIV, reproductive rights and informed choice and consent, safe sex and dual protection, values clarification, risk assessment and reduction, use of the BCS-plus, record keeping, logistics management and referral. Duration of the residential training was five days for the referral model and nine days for the testing model, which included an additional four days for training in HIV CT and conducting and interpreting rapid HIV tests. Trainers were drawn from DRH, NASCOP, AIDS \& STDs Coordinator and RH Training and Supervision Coordinator, Thika and Nyeri DHMTs, and

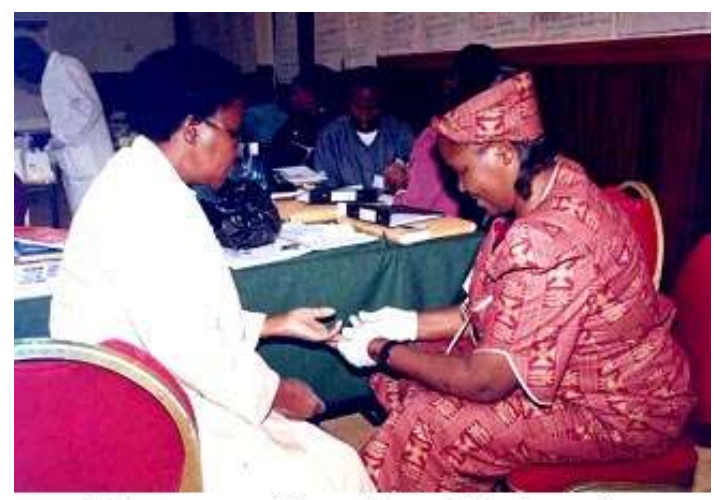

Trainees practicing taking a blood specimen FRONTIERS. To facilitate sustaining implementation and effective program supervision and monitoring, the national level trainers teamed up with provincial and district level trainers. Trainers followed up providers who had completed the training to ensure that they started implementation of activities immediately after training.

Participants took part in pre and post training tests. There was a marked improvement for all the groups in post training test scores (Table 1). The higher average score in Nyeri is attributed to the fact that providers in this district had recently undergone updates in FP under the "Implementing Best Practices (IBP)" pilot project a few months before the start of the training program.

\section{Table 1: Pre and post test mean scores for participants attending training sessions by district}

\begin{tabular}{|c|c|c|c|c|c|c|}
\hline \multicolumn{2}{|l|}{ District } & $\begin{array}{l}\text { Name of } \\
\text { group }\end{array}$ & $\begin{array}{l}\text { Group } \\
\text { size }\end{array}$ & $\begin{array}{c}\text { Pre-test } \\
\text { mean score }\end{array}$ & $\begin{array}{l}\text { Post-test } \\
\text { mean score }\end{array}$ & $\begin{array}{l}\% \text { increase in } \\
\text { mean scores }\end{array}$ \\
\hline \multirow[t]{2}{*}{ Nyeri } & \multirow{2}{*}{$\begin{array}{l}\text { April } 06 \\
\text { May } 06 \\
\end{array}$} & Group I & 12 & $63 \%$ & $84 \%$ & $33 \%$ \\
\hline & & Group II & 16 & $63 \%$ & $80 \%$ & $27 \%$ \\
\hline \multirow{2}{*}{ Thika } & \multirow{2}{*}{ May 06} & Group I & 22 & $56 \%$ & $73 \%$ & $30 \%$ \\
\hline & & Group II & 24 & $60 \%$ & $74 \%$ & $22 \%$ \\
\hline
\end{tabular}

Half of the participants rated the training to be very good, 30 percent it as good, while 18 percent rated the training as satisfactory, considering the following factors: content, materials and use of visual aids, trainers' presentation and practice sessions. Over half (58\%) of the participants said that the length of training was just right, while 42 percent thought it was too short. A number of participants recommended that the time allocated for the counseling and testing sessions should be increased to allow for more practice sessions.

Towards the end of the training, each participant developed an action plan that addressed preparatory steps and specific obstacles that needed to be tackled at their facility to be able to 
"fast-track" implementation of the activities. The action plans addressed five areas: identify problems; steps required to tackle the problems; the resources needed to tackle the problems; the persons responsible for tackling the problems and the expected initiation or completion dates for the planned activities.

\section{Strengthening basic supplies and commodities}

Planning for and procurement of key supplies, such as rapid test kits for HIV and reagents and FP commodities, was undertaken with district-level mechanisms for sourcing, storage, distribution and replenishment of supplies. NASCOP is responsible for provision of HIV test kits and the DRH for provision of FP commodities. In anticipation of starting up activities, both DHMTs were able to liaise with the PHMT, DRH and NASCOP, as well as the Kenya Medical Supplies Agency (KEMSA), to place orders for the requisite supplies. Study facilities embarked on implementation of activities immediately after receiving the basic supplies and commodities from the DHMTs.

\section{Strengthening routine data collection on FP and VCT services}

To ensure adequate monitoring of the intervention, the existing FP registers were reviewed. Additional information required to monitor implementation of the intervention was identified and key indicators (e.g. number of clients counseled, tested, referred and their HIV sero-status) were manually added to the registers in the intervention clinics before the new activities began. Collection, review and use of routine data from both FP and VCT registers were the key mechanism used to monitor implementation. Clinics and VCT centers were asked to keep records of the numbers of clients referred. The majority of health facilities in the referral model established systems for identifying the number of VCT clients who had been referred from FP clinics; examples included creation of additional columns in the register or inserting the letters "FP" against the names of clients who had been referred from FP clinics.

To better understand patterns of VCT utilization, clients in both models were given a set of VCT vouchers during their FP visit. The vouchers had two parts; the front part contained information about the referring institution, while the back side contained information about the receiving institution. Information on the referring institution included: name of district, name of issuing health facility/clinic, date of issue, whether client discussed with provider risk factors for STIs, HIV/AIDS and unintended pregnancy and whether client was new or repeat. Information on the receiving institution where the client went for the test included: date of test, name of testing health facility, whether the test was performed by FP provider or not, whether client received results or not, sex of the client and age in complete years. Once completed, the voucher was handed over to the midwife in charge of the FP clinic who then filed it. Information on the number of vouchers received was then compiled on monthly basis and handed over to the DHMT who then forwarded to the national level project team alongside information on other aspects of the project.

In both sites, activities were monitored and supervised using information collected from registers on FP and VCT utilization. In Thika district, additional information was collected on referrals. Project progress was reviewed on monthly basis by the respective DHMTs. Challenges arising were tackled at such meetings and during the monthly supportive supervision sessions. 


\section{EVALUATION METHODS}

\section{Study design and selection of sites}

A pre-post intervention design without a control group was used. Study participants were new and repeat FP clients and health providers (nurses and clinical officers) working in FP clinics. The study was conducted in two districts of Central Province, Nyeri and Thika, which represent examples of high and low availability of VCT services at the district level. At the time of designing the study, Nyeri District had three sites offering VCT while Thika District had 16 sites, mainly situated within existing health care facilities. The implementation phase lasted ten months, from May 2006 to February 2007.

Health facilities for piloting the two models were selected following consultations between the two relevant divisions of the $\mathrm{MOH}$, the Department of Reproductive Health (DRH) and the National AIDS and STI Control Program (NASCOP), the provincial and district health management teams and FRONTIERS. A total of 23 public health facilities were selected based on the following criteria:

- Adequate client volume (a minimum of 100 combined new and revisit clients per month);

- Minimum of two providers qualified in and currently providing FP services;

- Hospitals and health centers in Thika district were required to have VCT on site;

- Facilities in Nyeri district should not have VCT on site.

Of the 23 facilities selected, 14 implemented the referral model in Thika District and nine were selected to implement the testing model in Nyeri District. The selected facilities are listed in Appendix 4. In addition to fulfilling the criteria above, the DHMTs ensured that the selected facilities were evenly distributed across the administrative divisions to increase geographical coverage of health facilities. In Thika, the 14 sites included two hospitals, eight health centers and four dispensaries ${ }^{2}$. In Nyeri, the nine sites included one hospital, four health centers and four dispensaries.

\section{Health facility assessment and training audit}

A health facility assessment was conducted in all 23 facilities in March 2006 by experienced health providers who had been trained for two days to undertake the exercise. The assessment involved a review of records, interview with health facility in-charges, and an inventory of equipment and supplies available for providing FP and counseling and testing services. A training audit was also undertaken to assess the number and skills of health staff available.

\section{Focus group discussions with FP providers}

Pre and post-intervention FGDs were conducted in March 2006 and in April 2007 to assess the acceptability of the interventions to providers in both districts. One group of health providers from each district participated in the FGDs. In the pre-intervention FGDs, providers were

2 A dispensary usually offers a limited range of curative, preventive, promotive and rehabilitative care. On average, dispensaries are staffed with 2-3 nurses. Dispensaries often lack a laboratory, maternity ward, inpatient facilities and staff houses. A health centre (with an average staff of 5-8 health providers) offers a wider range of services, including a laboratory, maternity services, inpatient facilities etc. 
selected from non-study facilities, whereas providers in the post-intervention FGDs were selected from all the study sites. The FGDs were carried out by two social scientists with the help of a reproductive health trainer from the $\mathrm{MOH}$. Providers were asked to comment on challenges in providing $\mathrm{FP}$, their perceptions of the quality of care, current provision of services, contribution of FP services in combating HIV and STIs, cost of FP/STI services, and the acceptability of integrating counseling and testing services for HIV within FP services. The characteristics of health providers who participated in the discussions were similar at baseline and endline.

\section{Focus group discussions with FP clients}

FGDs with FP clients were held in each district (one at a health center and one at a dispensary) before and after the interventions. Participants in the pre-intervention FGDs were selected from clients attending FP services in four randomly selected non-study facilities. Post-intervention FGDs were held in four facilities among those that had implemented the interventions, two in Nyeri and two in Thika. Clients were asked to comment on the current challenges to provision of FP care, their perception on the quality of care, access to VCT services, factors affecting VCT uptake and access and the acceptability of integrating counseling and testing services for HIV within FP services.

\section{Observations of provider-client interaction}

In each of the 23 facilities, a trained nurse observed approximately 24 consecutive new and repeat FP client-provider interactions. A total of 554 observations were completed at baseline (April 2006) and 530 at endline (March 2007) (see Table 2). During the observation, the nurse used a structured checklist with a series of standard items to record the technical competence of the provider as well as the quality of care received.

\section{Client exit interviews}

Clients exiting the observed FP consultations were then requested to be interviewed by trained research assistants using a structured questionnaire. A total of $552 \mathrm{FP}$ client exit interviews were completed at baseline and $530 \mathrm{FP}$ interviews completed at endline (see Table 2). Clients were asked questions relating to the acceptability and quality of services they had just received and their experiences with testing for HIV or intention to test, dual protection, behavior during the period, experience of the service, changes in risk perception, and partner notification and testing.

Table 2: Sample sizes for client-provider observations and client exit interviews

\begin{tabular}{|c|c|c|c|}
\hline \multicolumn{2}{|c|}{} & \multicolumn{2}{|c|}{ Number of clients } \\
\cline { 3 - 4 } \multicolumn{2}{|c|}{} & Baseline & Endline \\
\hline \multirow{2}{*}{ Client-provider observations } & Thika & 354 & 317 \\
\cline { 2 - 4 } & Nyeri & 200 & 213 \\
\hline \multirow{2}{*}{ Client exit interviews } & Thika & 346 & 316 \\
\cline { 2 - 4 } & Nyeri & 206 & 214 \\
\hline
\end{tabular}




\section{Measuring incremental costs of implementing each model}

The costing framework for integrating CT for HIV into FP services took into account only the additional costs incurred for integrating the additional services; see Appendix six for a detailed description of the methodology used. The incremental costs considered in this report are confined to the service delivery phase only, although the report also shows the costs incurred during the planning phase and training phases. Retrospective data (pre-intervention) and prospective data (post-intervention) were collected from the 23 facilities. Data were obtained from: key informant interviews, review of administrative data, FP utilization registers and observation of the time each provider spent with clients. During the health facility assessment, information on costing was obtained from facility in-charges and KEMSA on HIV equipment and supplies. Costing information covered the quantities of supplies used before and during intervention period. For each item, the price per unit of pack or quantities used was obtained. The total quantities used were multiplied by the respective unit cost of the supplies used to obtain the total cost of the various interventions.

Information on labor costs was obtained by reviewing employment details of the staff observed during client-provider interactions. Details included job title, days worked per year, hours worked per year, basic monthly gross salary and monthly allowances for housing, medical, leave, non-practice, hardship and other monthly benefits.

\section{Data management and analysis}

Data entry screens for all quantitative data were set up in Epidata and double entered. Analysis was done in Stata 9.2. Client characteristics were compared at baseline and any differences observed were adjusted for when making comparisons post-intervention. Qualitative data were analyzed manually using content analysis techniques. Ethical considerations including informed consent for study participants, informed consent for VCT clients and issues pertaining to confidentiality, among others, were observed and adhered to throughout the study.

\section{FINDINGS}

\section{Readiness to offer integrated services}

At baseline, the majority of facilities had the minimum basic equipment, supplies, infrastructure and staff to carry out integration activities in both models (Table 3 and Appendix 5). The results are expressed in terms of mean percentage scores and were derived from individual percentage scores against all the items listed under each category in Appendix 5. There were variations; for instance, whereas all facilities had BP machines, stethoscopes, and examination couches, other equipment such as port light, display board for FP methods, sterilizing drums were only available in approximately $50 \%$ of the facilities across the two models. Overall, clinics implementing the testing model had a slightly higher mean readiness score (74\%) than those in the referral model (68\%). As shown in Appendix 5, although most facilities had short-term FP methods available, they were likely to have had stock outs for implants, emergency contraceptives, and IUDs. In addition, provision of surgical contraception (female mini laparotomy, tubal ligation and vasectomy for males) was quite low, especially in Thika District. 
Table 3: Availability of basic equipment, supplies, infrastructure and services for FP and HIV/STIs

\begin{tabular}{|l|c|c|c|}
\hline Category & $\begin{array}{c}\text { Testing model } \\
\mathbf{n = 9}(\%)\end{array}$ & $\begin{array}{c}\text { Referral model } \\
\mathbf{n = 1 4}(\%)\end{array}$ & $\begin{array}{c}\text { Pooled } \\
\mathbf{n = 2 3}(\%)\end{array}$ \\
\hline Availability of equipment & 76 & 74 & 74 \\
\hline Availability of FP supplies & 52 & 43 & 46 \\
\hline Availability of HIV/STI supplies & 81 & 80 & 81 \\
\hline Availability of infrastructure & 83 & 84 & 84 \\
\hline Provision of FP services & 73 & 56 & 63 \\
\hline Provision of HIV/STI services & 77 & 71 & 70 \\
\hline Overall mean Score & 74 & 68 & 6 \\
\hline Staff & \multicolumn{3}{|c|}{7} \\
\hline $\begin{array}{l}\text { Average No. of Enrolled nurses / } \\
\text { midwives working in the MCH/FP unit }\end{array}$ & 4 & 1 & 1 \\
\hline $\begin{array}{l}\text { Average number of Registered } \\
\text { nurses/ midwives in MCH/FP unit }\end{array}$ & 1 & 7 & \multirow{2}{*}{} \\
\hline
\end{tabular}

\section{Acceptability of services to clients}

Comments by clients at the endline about the quality of FP services suggests that there was improvement over the intervention period as there were fewer complaints, especially about the shortage of FP commodities. Despite the improvements, clients at the endline made suggestions for further improvements:

"Would like to see additional providers due to high number of clients" Client, Nyeri District.

"FP commodities stock-outs cause some clients to switch methods” Client, Thika District.

"FP services should be made free of charge since some clients are not able to meet the cost of these FP services and this is a service all women require." Client, Thika District.

Respondents, especially in Thika district, said that they were told where to get VCT services and also received information from their provider on VCT services in general, such as the importance of knowing own HIV status. In both districts, there was a marked reduction in the number of clients at endline who mentioned stigma and self-denial as issues that discourage people from going for VCT. At endline, clients appeared more concerned about meeting the needs of discordant couples: "People are discouraged from going for testing because if one partner is positive and the other negative it causes separation." (Client, Thika district). The majority of participants in the testing model felt that the intervention improved access, as they were not required to travel to other health facilities to be counseled and tested for HIV.

During the baseline, the majority of clients expressed a willingness to go for VCT services. Some of them preferred attending VCT in far and secluded places away from their homes. However, at endline, more clients (especially in the testing model) preferred to be tested in the same health facility as for FP services. Reasons included time saving and that only one provider need know their status. Many clients stated (at baseline and endline) that the availability of ARVs could alter the community's perception and make more people go for VCT services.

Overall, participants said that it was a good idea to integrate counseling and testing within FP services. They liked the idea of the provider knowing their status and guiding them if positive on appropriate FP methods. However, there were mixed reactions about being referred elsewhere 
for VCT services by an FP provider. Some felt that it was acceptable if the provider gives good reasons for referral. However, majority said they would prefer to be tested at their local clinic together with their partners.

"It is better for my FP provider to test me and know my status. I do not mind about the provider knowing my status so long as I get the services I require" Client, Nyeri District.

"It is a good idea to receive both counseling and testing and a method from our FP clinic"...

if nurse knows our HIV status may guide us if positive on FP methods" Client, Thika District.

Exit interviews with FP clients revealed that integrating counseling and testing for HIV within family planning services was acceptable, with over 90 percent of all clients expressing satisfaction with the service received.

\section{Acceptability to providers}

Most providers were female and the mean age was 46 years in Nyeri District and 43 years in Thika District. At baseline, the main challenges cited by providers were irregular supply of FP commodities and shortage of staff in health facilities. At endline, the challenges mentioned were more to do with the effects of implementation of integrated activities. These included increased waiting time for clients as consultations for integrated services take longer, difficulties in using the BCS-plus job aids, and a small number of partner vouchers returned to the facilities.

"Due to shortage of staff and the added HIV services the workload is high and this forces us to hold clients for long, which then causes a lot of complaints from them." Provider, Thika District.

In both districts, providers suggested that the $\mathrm{MOH}$ deploys additional providers to address the issue of staff shortages. Providers also pointed out that although they are trying to integrate HIV and FP services, some of them do not have skills in counseling and testing as well as in the broader management of HIV positive clients, especially those requiring specific drugs:

"Clients on ARVS and TB are difficult to manage, especially if they have been on TB drugs and test HIV positive. In such cases, we do not know which drug to start on." Provider, Thika District.

Providers indicated that they had adopted a number of strategies and coping mechanisms to maintain the quality of FP care provided to clients because of the shortage of commodities and human resources. In both districts, providers indicated that they reduce the VCT/FP consultation time by doing group counseling for pre-test counseling and only do individual counseling for the post-test counseling. In both districts, providers said that integrating counseling and testing for HIV within FP services has enabled many clients to access HIV/AIDS/STIs services and care, especially those who might have feared going to VCT sites. Condom use in particular is encouraged.

"We encourage those who test positive that they do not have to entirely give up their sex lives, but to strictly ensure that they use condoms to avoid re-infections and transmission of the virus." Provider, Nyeri District 
In both districts, providers indicated that the cost of accessing the two services was low enough to not prevent would-be clients from seeking the services. They said the use of the syndromic approach in managing FP clients with RTIs/STIs helps to lower the cost of providing services since laboratory tests are not required.

Overall, providers felt that it is feasible and acceptable to integrate counseling and testing for HIV within family planning services. The majority of providers felt that provision of integrated services was beneficial to both themselves and their clients. They also felt that integration had improved client-provider interactions, couple counseling, and male involvement in VCT/FP issues. Providers in Nyeri said that the testing model had empowered them with appropriate skills to counsel and test clients for HIV. Providers in referral model clinics in Thika recommended that the $\mathrm{MOH}$ allows them to have onsite counseling and testing services for HIV.

\section{Characteristics of the FP clients}

Table 4: Characteristics of clients by model

\begin{tabular}{|c|c|c|c|c|}
\hline & \multicolumn{2}{|c|}{ Testing model (Nyeri) } & \multicolumn{2}{|c|}{ Referral model (Thika) } \\
\hline & $\begin{array}{c}\text { Baseline } \\
\mathrm{n}=214\end{array}$ & $\begin{array}{c}\text { Endline } \\
\mathrm{n}=210\end{array}$ & $\begin{array}{c}\text { Baseline } \\
n=324\end{array}$ & $\begin{array}{c}\text { Endline } \\
n=310\end{array}$ \\
\hline \multicolumn{5}{|c|}{ Demographic and fertility indicators (from client exit interview): } \\
\hline Age $\leq 20$ years & 4 & 5 & 4 & 5 \\
\hline Age $21-30$ years & 45 & 50 & 55 & 56 \\
\hline Age $31-40$ years & 38 & 37 & 31 & 30 \\
\hline Age $\geq 41$ years & 13 & 9 & 11 & 9 \\
\hline Median age & $31 \mathrm{yrs}$ & $29 \mathrm{yrs}$ & $29 \mathrm{yrs}$ & $28 \mathrm{yrs}$ \\
\hline Lower primary school (1-4) and none & 5 & 8 & 11 & 9 \\
\hline Completed upper primary school $(5-8)$ & 70 & 64 & 63 & 68 \\
\hline Completed Secondary School and above & 25 & 28 & 26 & 23 \\
\hline Married/Cohabiting & 96 & 94 & 95 & 96 \\
\hline Median number living children & 3 & 2 & 2 & 3 \\
\hline & & & & \\
\hline Want more children & 37 & 36 & 43 & 41 \\
\hline Do not want more children & 58 & 59 & 50 & 58 \\
\hline Uncertain & 5 & 5 & 3 & 1 \\
\hline Of those wanting more, want within $1-2$ years & 23 & 25 & 13 & 10 \\
\hline \multicolumn{5}{|c|}{ Purpose and outcome of visit (from client-provider observation) } \\
\hline New user of FP method & 14 & 13 & 19 & 16 \\
\hline Repeat user of FP method & 86 & 87 & 81 & 84 \\
\hline \multicolumn{5}{|l|}{ FP method used on day of visit } \\
\hline Injectable & 71 & 79 & 69 & 77 \\
\hline Pill (any type) & 23 & 17 & 26 & 4 \\
\hline Condom & 1 & 2 & 1 & 18 \\
\hline IUD & 4 & 3 & 1 & 2 \\
\hline Others & 1 & 0 & 3 & 0 \\
\hline
\end{tabular}


As can be seen in Table 4, most clients were married, around 30 years old, had two or three living children, and had completed $5-8$ years of schooling. There were some differences between the districts in terms of fertility intentions, with those in Nyeri less likely to want more children, and for those who did want another child, women in Nyeri were more likely to want to deliver within two years. Between 13 - 19 percent of clients were 'new', that is obtaining family planning from the clinic for the first time. Reflecting the national situation in Kenya, the vast majority of clients were using hormonal contraception, mostly injectables. This is despite the fact that well over half of the FP clients do not want any more children at all, and of those that do want more, 80 percent would like to wait at least three years. The low levels of IUD use, a method well-suited to this population, also reflect national norms.

\section{Quality of FP Counseling}

After updating providers in FP and orienting them in the BCS-plus approach, significant improvements were observed in FP method counseling in both models across most of the six indicators that were assessed (see table 5). These indicators were chosen as they represent the key issues usually considered necessary for good quality of counseling on family planning (e.g. Bruce 1990). To assess the magnitude of overall changes in the quality of FP counseling within and between the two models, summary scores were developed by aggregating the mean scores of the six indicators for each individual observed, giving a scale ranging from 0 (lowest quality) to 6 (highest quality).

Table 5: Proportions of consultations in which FP issues were discussed

\begin{tabular}{|c|c|c|c|c|c|c|c|}
\hline \multirow{3}{*}{\multicolumn{2}{|c|}{$\begin{array}{l}\text { Proportion of consultations in } \\
\text { which provider: }\end{array}$}} & \multicolumn{2}{|c|}{$\begin{array}{l}\text { Testing Model } \\
\text { (Nyeri) }\end{array}$} & \multicolumn{2}{|c|}{$\begin{array}{l}\text { Referral Model } \\
\text { (Thika) }\end{array}$} & \multicolumn{2}{|c|}{ Pooled Sample } \\
\hline & & \multirow{2}{*}{$\begin{array}{c}\text { Baseline } \\
(\mathrm{N}=214) \\
\%\end{array}$} & \multirow{2}{*}{$\begin{array}{c}\text { Endline } \\
\text { (N=210) } \\
\%\end{array}$} & \multirow{2}{*}{$\begin{array}{c}\text { Baseline } \\
(\mathrm{N}=324) \\
\%\end{array}$} & \multirow{2}{*}{$\begin{array}{c}\text { Endline } \\
\text { (N=310) } \\
\%\end{array}$} & \multirow{2}{*}{$\begin{array}{c}\text { Baseline } \\
\left(\begin{array}{c}N=538) \\
\%\end{array}\right.\end{array}$} & \multirow{2}{*}{$\begin{array}{c}\text { Endline } \\
\begin{array}{c}\mathrm{N}=520) \\
\%\end{array}\end{array}$} \\
\hline & & & & & & & \\
\hline 1 & $\begin{array}{l}\text { Discussed reproductive } \\
\text { intentions }\end{array}$ & 13 & 14 & 15 & $40^{* *}$ & 14 & $30^{* *}$ \\
\hline 2 & $\begin{array}{l}\text { Discussed previous use of } \\
\text { FP }\end{array}$ & 66 & $79^{* *}$ & 66 & $78^{* *}$ & 66 & $79^{* *}$ \\
\hline 3 & $\begin{array}{l}\text { Discussed } 2 \text { or more } \\
\text { methods }\end{array}$ & 43 & $53^{*}$ & 45 & $86^{* *}$ & 44 & $73^{* *}$ \\
\hline 4 & $\begin{array}{l}\text { Provided with choice } \\
\text { regarding preferred method }\end{array}$ & 76 & $95^{* *}$ & 92 & 93 & 85 & $93^{* *}$ \\
\hline 5 & $\begin{array}{l}\text { Discussed how chosen } \\
\text { method works }\end{array}$ & 34 & 42 & 54 & $78^{* *}$ & 46 & $64^{\star *}$ \\
\hline 6 & $\begin{array}{l}\text { Explained advantages/ } \\
\text { disadvantages of chosen } \\
\text { method }\end{array}$ & 25 & $48^{* *}$ & 50 & $77^{* *}$ & 40 & $65^{* *}$ \\
\hline \multicolumn{2}{|r|}{ Total Score $(0-6)$} & 2.52 & $3.23^{* \star}$ & 3.16 & $4.41^{\star *}$ & 2.91 & $3.93^{\star \star}$ \\
\hline
\end{tabular}

${ }^{*}$ Significant at $p<0.05 ;{ }^{* *} \mathrm{P}<0.01$

The summary scores show that the increase in quality was higher in the referral model clinics, although these did start at a higher level of quality than the testing model clinics. The greater increase in this group may be due to the fact that providers in the referral model clinics (Thika district) were much more likely (75\%) to have been observed using the BCS-plus approach during consultations than providers in the testing model clinics (41\%) (Nyeri district), supporting 
findings from earlier studies that the BCS-plus does have an effect on quality of FP counseling. Overall, the pooled sample shows that use of the BCS-Plus increased mean number of critical actions from approximately three to four per consultation. The weakest aspect remained discussions of fertility intentions, with providing clients with a choice of methods consistently being the strongest aspect.

Significant improvements were also noted with regard to the rapport established between provider and client (see table 6), although interestingly the improvements were greater in the testing model clinics than the referral model clinics. Some indicators (e.g. privacy and the use of client records during consultation; not included here) did not improve, but were already high at baseline.

One of the biggest improvements was in the proportion of consultations in which the provider spent more than 15 minutes with their client. At baseline, the median consultation time for clients attending the referral and testing models was 12 and 10 minutes respectively; at endline, this had increased to 15 and 14 minutes respectively, regardless of whether the clients were repeat or new. The median consultation time for new clients at endline was 18 minutes in the referral model and 20 minutes in the testing model. The median consultation time for all clients in the testing model (repeat or new) who were actually tested was 17 minutes, which means that the addition of the test increases the consultation time by an average of seven minutes.

Table 6: Proportion of consultations in which client rapport established

\begin{tabular}{|c|c|c|c|c|c|c|c|}
\hline \multirow{2}{*}{\multicolumn{2}{|c|}{$\begin{array}{l}\text { Proportion of consultations in } \\
\text { which provider: }\end{array}$}} & \multicolumn{2}{|c|}{$\begin{array}{l}\text { Testing Model } \\
\text { (Nyeri) }\end{array}$} & \multicolumn{2}{|c|}{$\begin{array}{l}\text { Referral Model } \\
\text { (Thika) }\end{array}$} & \multicolumn{2}{|c|}{ Pooled Sample } \\
\hline & & \multirow{2}{*}{$\begin{array}{c}\text { Baseline } \\
(\mathrm{N}=214) \\
\%\end{array}$} & \multirow{2}{*}{$\begin{array}{c}\text { Endline } \\
\begin{array}{c}\mathrm{N}=210) \\
\%\end{array}\end{array}$} & \multirow{2}{*}{$\begin{array}{c}\text { Baseline } \\
(\mathrm{N}=324) \\
\%\end{array}$} & \multirow{2}{*}{$\begin{array}{c}\text { Endline } \\
(\mathrm{N}=310) \\
\%\end{array}$} & \multirow{2}{*}{$\begin{array}{c}\text { Baseline } \\
(\mathrm{N}=538) \\
\%\end{array}$} & \multirow{2}{*}{$\begin{array}{c}\text { Endline } \\
(\mathrm{N}=520) \\
\%\end{array}$} \\
\hline & & & & & & & \\
\hline 1 & Client greeted warmly & 88 & $95^{\star *}$ & 85 & $94^{* *}$ & 86 & $94^{* *}$ \\
\hline 2 & Discussed medical conditions & 33 & $59^{* *}$ & 54 & 40 & 46 & 48 \\
\hline 3 & $\begin{array}{l}\text { Asked if client understood } \\
\text { information }\end{array}$ & 74 & $89^{* *}$ & 83 & $90^{* *}$ & 79 & $89^{* *}$ \\
\hline 4 & $\begin{array}{l}\text { Encouraged client to ask } \\
\text { questions }\end{array}$ & 67 & $94^{* *}$ & 71 & $88^{* *}$ & 69 & $90^{* *}$ \\
\hline 5 & Used client's name & 61 & $72^{*}$ & 56 & 63 & 58 & $67^{\star *}$ \\
\hline 6 & Help in decision-making & 53 & $77^{* *}$ & 85 & 85 & 73 & $82^{* *}$ \\
\hline 7 & Consultation time $>15$ minutes & 29 & $40^{* *}$ & 37 & $48^{* *}$ & 33 & $45^{\star *}$ \\
\hline \multicolumn{2}{|c|}{ Total Score (0-7) } & 4.05 & $5.24^{\star \star}$ & 4.71 & $5.02^{*}$ & 4.44 & $5.11^{\star \star}$ \\
\hline
\end{tabular}

${ }^{*}$ Significant at $\mathrm{p}<0.05 ;{ }^{* *} \mathrm{P}<0.01$

\section{Quality of STI/HIV Counseling}

Overall, improvements in the quality of counseling for STI/HIV risk factors were observed in both models for the set of factors described in Table 7, but the low summary scores of around two out of five key actions being undertaken with each client after the intervention indicate that this is an area that needs more attention. 
Table 7: Discussion of STI/HIV issues with FP clients

\begin{tabular}{|c|c|c|c|c|c|c|c|}
\hline \multirow{3}{*}{\multicolumn{2}{|c|}{$\begin{array}{l}\text { Proportion of consultations in } \\
\text { which provider: }\end{array}$}} & \multicolumn{2}{|c|}{$\begin{array}{c}\text { Testing Model } \\
\text { (Nyeri) }\end{array}$} & \multicolumn{2}{|c|}{$\begin{array}{l}\text { Referral Model } \\
\text { (Thika) }\end{array}$} & \multicolumn{2}{|c|}{ Pooled Sample } \\
\hline & & \multirow{2}{*}{$\begin{array}{c}\text { Baseline } \\
(\mathrm{N}=214) \\
\%\end{array}$} & \multirow{2}{*}{$\begin{array}{c}\text { End line } \\
\begin{array}{c}\mathrm{N}=210) \\
\%\end{array}\end{array}$} & \multirow{2}{*}{$\begin{array}{c}\text { Baseline } \\
(\mathrm{N}=324) \\
\%\end{array}$} & \multirow{2}{*}{$\begin{array}{c}\text { Endline } \\
(\mathrm{N}=310) \\
\%\end{array}$} & \multirow{2}{*}{$\begin{array}{c}\text { Baseline } \\
(\mathrm{N}=538) \\
\%\end{array}$} & \multirow{2}{*}{$\begin{array}{c}\text { Endline } \\
\begin{array}{c}\mathrm{N}=520) \\
\%\end{array}\end{array}$} \\
\hline & & & & & & & \\
\hline 1 & $\begin{array}{l}\text { Discussed client history of STI } \\
\text { symptoms }\end{array}$ & 18 & 13 & 11 & $38^{* *}$ & 14 & $28^{* *}$ \\
\hline 2 & $\begin{array}{l}\text { Discussed number of sexual } \\
\text { partners }\end{array}$ & 5 & $11^{*}$ & 1 & $19^{* *}$ & 3 & $16^{\star *}$ \\
\hline 3 & Discussed STI/HIV/AIDS & 48 & $84^{* *}$ & 30 & $77^{\star *}$ & 37 & $80^{* *}$ \\
\hline 4 & $\begin{array}{l}\text { Discussed STI/HIV/AIDS risk } \\
\text { factors }\end{array}$ & 39 & $52^{* *}$ & 22 & $69^{* *}$ & 29 & $62^{* *}$ \\
\hline 5 & $\begin{array}{l}\text { Tells client STI increase risk } \\
\text { of HIV }\end{array}$ & 14 & $7^{*}$ & 20 & $43^{\star *}$ & 17 & $28^{* *}$ \\
\hline \multicolumn{2}{|c|}{ Total Score (0-5) } & 1.24 & $1.68^{\star *}$ & 0.83 & $2.46^{\star \star}$ & 1.00 & $2.14^{\star \star}$ \\
\hline
\end{tabular}

* Significant at $p<0.05 ;{ }^{* *} P<0.01$

Substantial improvements were made in the clinics in Thika district, which may reflect greater use of the BCS-Plus approach, as noted above. Providers seem to be most comfortable having general discussions about STIs/HIV and to a lesser extent discussing some of the risk factors, but counseling about the client's personal behaviors (e.g. history of symptoms or number of sexual partners), remains extremely low. This demonstrates once again the often observed reluctance of FP providers to engage their clients in discussions of their personal sexual behavior.

\section{Quality of counseling about condom use}

As shown in Table 8 , all indicators relating to counseling on dual protection improved significantly after introducing the BCS-Plus approach, and particularly in the referral model clinics in Thika district. At baseline, this had been a particularly weak aspect of counseling, with average summary scores of 0.51 and 0.8 items per client. Although there is still much improvement needed, the increase to 2.4 items per client on average, including 2.88 in Thika district, indicate that providers are willing and able to discuss condom use in a dual protection perspective with FP clients.

In most facilities, condoms have been placed in dispensers and clients are expected to take themselves from these dispensers. The proportion of consultations in which a provider was observed giving male condoms to clients improved significantly after the intervention, however, from two percent at baseline to eleven percent at endline; there was also a slight increase in the provision of female condoms from none at all at baseline to two percent of consultations at endline. These proportions were higher in the referral model. 
Table 8: Counseling and provision of condoms

\begin{tabular}{|c|c|c|c|c|c|c|c|}
\hline \multirow{3}{*}{\multicolumn{2}{|c|}{$\begin{array}{l}\text { Proportion of consultations in } \\
\text { which provider: }\end{array}$}} & \multicolumn{2}{|c|}{$\begin{array}{c}\text { Testing Model } \\
\text { (Nyeri) }\end{array}$} & \multicolumn{2}{|c|}{$\begin{array}{l}\text { Referral Model } \\
\text { (Thika) }\end{array}$} & \multicolumn{2}{|c|}{ Pooled Sample } \\
\hline & & \multirow{2}{*}{$\begin{array}{c}\text { Baseline } \\
(\mathrm{N}=214) \\
\%\end{array}$} & \multirow{2}{*}{$\begin{array}{c}\text { Endline } \\
\begin{array}{c}\mathrm{N}=210) \\
\%\end{array}\end{array}$} & \multirow{2}{*}{$\begin{array}{c}\text { Baseline } \\
(\mathrm{N}=324) \\
\%\end{array}$} & \multirow{2}{*}{$\begin{array}{c}\text { Endline } \\
(\mathrm{N}=310) \\
\%\end{array}$} & \multirow{2}{*}{$\begin{array}{c}\text { Baseline } \\
(\mathrm{N}=538) \\
\%\end{array}$} & \multirow{2}{*}{$\begin{array}{c}\text { Endline } \\
\begin{array}{c}\mathrm{N}=520) \\
\%\end{array} \\
\end{array}$} \\
\hline & & & & & & & \\
\hline 1 & $\begin{array}{l}\text { Explains condoms protect against } \\
\text { STIs/HIV and pregnancy }\end{array}$ & 22 & $33^{* *}$ & 11 & $57^{* *}$ & 16 & $48^{* *}$ \\
\hline 2 & $\begin{array}{l}\text { Encourages use of condoms with } \\
\text { another FP method }\end{array}$ & 32 & $50^{* *}$ & 12 & $71^{* *}$ & 20 & $62^{* *}$ \\
\hline 3 & Explains how to use a condom & 9 & $33^{* *}$ & 12 & $62^{* *}$ & 11 & $51^{* *}$ \\
\hline 4 & $\begin{array}{l}\text { Emphasize correct/consistent } \\
\text { condom use }\end{array}$ & 10 & $30^{* *}$ & 9 & $53^{* *}$ & 9 & $43^{* *}$ \\
\hline 5 & $\begin{array}{l}\text { Explains how to negotiate condom } \\
\text { use }\end{array}$ & 8 & $23^{* *}$ & 7 & $46^{* *}$ & 7 & $37^{* *}$ \\
\hline \multicolumn{2}{|r|}{ Total Score (0-5) } & 0.80 & $1.70^{\star *}$ & 0.51 & $2.88^{* *}$ & 0.63 & $2.40^{\star \star}$ \\
\hline
\end{tabular}

* Significant at $p<0.05 ;{ }^{* *} \mathrm{P}<0.01$

\section{Quality of counselling on HIV C\&T}

Training, encouraging and supplying job aides for providers to discuss HIV CT with their FP clients has clearly had a substantial effect on their consultations. As Table 9 shows, the summary scores increased from approximately one item to almost three out of four items per client. After the intervention, counseling and testing for HIV was mentioned in almost 90 percent of consultations overall, and the client's own serostatus was discussed.

Table 9: Counseling on CT services

\begin{tabular}{|c|c|c|c|c|c|c|c|}
\hline \multirow{3}{*}{\multicolumn{2}{|c|}{$\begin{array}{l}\text { Proportion of consultations in } \\
\text { which provider: }\end{array}$}} & \multicolumn{2}{|c|}{$\begin{array}{l}\text { Testing Model } \\
\text { (Nyeri) }\end{array}$} & \multicolumn{2}{|c|}{$\begin{array}{l}\text { Referral Model } \\
\text { (Thika) }\end{array}$} & \multicolumn{2}{|c|}{ Pooled Sample } \\
\hline & & \multirow{2}{*}{$\begin{array}{c}\text { Baseline } \\
\begin{array}{c}\mathrm{N}=214) \\
\%\end{array} \\
\end{array}$} & \multirow{2}{*}{$\begin{array}{c}\text { End line } \\
\begin{array}{c}\mathrm{N}=210) \\
\%\end{array} \\
\end{array}$} & \multirow{2}{*}{$\begin{array}{c}\text { Baseline } \\
(\mathrm{N}=324) \\
\%\end{array}$} & \multirow{2}{*}{$\begin{array}{c}\text { Endline } \\
\left(\begin{array}{c}N=310) \\
\%\end{array}\right. \\
\end{array}$} & \multirow{2}{*}{$\begin{array}{c}\text { Baseline } \\
(\mathrm{N}=538) \\
\%\end{array}$} & \multirow{2}{*}{$\begin{array}{c}\text { Endline } \\
\text { (N=520) } \\
\%\end{array}$} \\
\hline & & & & & & & \\
\hline 1 & Discuss HIV serostatus & 30 & $77^{\star \star}$ & 20 & $83^{* *}$ & 24 & $81^{* *}$ \\
\hline 2 & Mentions VCT & 42 & $82^{* *}$ & 37 & $92^{* *}$ & 39 & $88^{* *}$ \\
\hline 3 & $\begin{array}{l}\text { Discuss what test tells } \\
\text { client }\end{array}$ & 36 & $66^{* *}$ & 31 & $57^{* *}$ & 33 & $61^{* *}$ \\
\hline 4 & $\begin{array}{l}\text { Explain about window } \\
\text { period }\end{array}$ & 1 & $53^{* *}$ & 7 & $31^{* *}$ & 5 & $40^{* *}$ \\
\hline \multicolumn{2}{|c|}{ Total Score (0-4) } & 1.10 & $2.78^{\star \star}$ & 0.95 & $2.64^{\star \star}$ & 1.01 & $2.70^{\star *}$ \\
\hline
\end{tabular}

${ }^{*}$ Significant at $\mathrm{p}<0.05 ;{ }^{* *} \mathrm{P}<0.01$

\section{Aggregate scores for quality of care}

To evaluate the overall effect of the introduction of the BCS-Plus approach on the quality of care received in both the testing and referral models, Table 10 summarizes the mean scores for each of the components assessed and presents a summary aggregate score for each model / district and 
for the total sample. At baseline, providers were observed counseling FP clients on approximately ten out of the 27 items selected to measure quality of care. At the time of the endline, this had increased significantly, to almost 15 items in the Nyeri testing model clinics and to almost 17.5 items in the Thika referral model clinics.

Table 10: Summary scores for quality of care components before and after intervention

\begin{tabular}{|c|c|c|c|c|c|c|}
\hline \multirow{3}{*}{ Quality of care components } & \multicolumn{2}{|c|}{$\begin{array}{c}\begin{array}{c}\text { Testing Model } \\
\text { (Nyeri) }\end{array} \\
\end{array}$} & \multicolumn{2}{|c|}{ Referral Model (Thika) } & \multicolumn{2}{|c|}{ Pooled Sample } \\
\hline & Baseline & Endline & Baseline & Endline & Baseline & Endline \\
\hline & $(\mathrm{N}=214)$ & $(\mathrm{N}=210)$ & $(\mathrm{N}=324)$ & $(\mathrm{N}=310)$ & $(\mathrm{N}=538)$ & $(\mathrm{N}=520)$ \\
\hline FP method counseling (0-6) & 2.52 & $3.23^{* *}$ & 3.16 & $4.41^{* *}$ & 2.91 & $3.93^{* *}$ \\
\hline Client - provider rapport (0-7) & 4.05 & $5.24^{* *}$ & 4.71 & $5.02^{*}$ & 4.44 & $5.11^{* *}$ \\
\hline STI prevention counseling $(0-5)$ & 1.24 & $1.68^{* *}$ & 0.83 & $2.46^{* *}$ & 1.00 & $2.14^{* *}$ \\
\hline Dual protection counseling (0-5) & 0.80 & $1.70^{* *}$ & 0.51 & $2.88^{* *}$ & 0.63 & $2.40^{* *}$ \\
\hline VCT counseling $(0-4)$ & 1.10 & $2.78^{* *}$ & 0.95 & $2.64^{* *}$ & 1.01 & $2.70^{* *}$ \\
\hline Total score $(0-27)$ & 9.71 & $14.63^{\star \star}$ & 10.16 & $17.41^{\star \star}$ & 9.99 & $16.28^{\star \star}$ \\
\hline
\end{tabular}

* Significant at $\mathrm{p}<0.05 ; * * \mathrm{P}<0.01$

As noted above, this difference between the models / districts may be due to the much higher levels of use of the BCS-Plus approach in Thika district. To explore this possibility further, the scores for those consultations in which the BCS-Plus approach was used were compared with the scores for consultations in which the approach was not used. As Figure 2 shows, the summary scores for all consultations when the BCS Plus approach is used are much higher than when it is not used, clearly indicating that this approach is an effective means for improving the quality of counseling when providing integrated services. The approach was particularly effective in Thika district, causing a doubling in the quality of care scores.

Figure 2: Summary measures of quality of counseling by use or non-use of the BCS Plus

Score $0-27$

$\square$ Not use BCS+ $\square$ Use BCS+

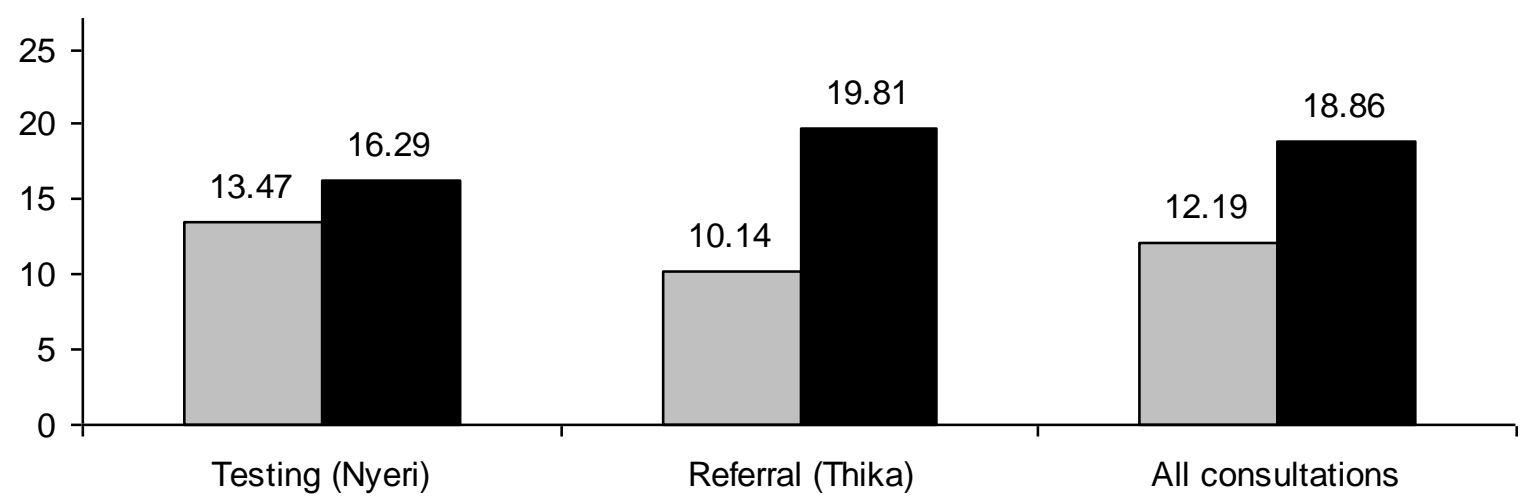




\section{Effect on condom use}

As Table 11 shows, although remaining low overall, recent and current condom use increased significantly among most clients. Of particular note are the small but significant increases in the proportions of FP clients using condoms jointly with another FP method; this is particularly marked among repeat clients, which may be attributable to messages given during use of the BCS Plus.

Table 11: Clients' use of condoms (percentages)

\begin{tabular}{|l|c|c|c|c|}
\hline \multirow{2}{*}{ Proportion of FP clients who: } & \multicolumn{2}{|c|}{$\begin{array}{c}\text { Testing Model } \\
\text { (Nyeri) }\end{array}$} & \multicolumn{2}{c|}{ Referral Model (Thika) } \\
\cline { 2 - 5 } & $\begin{array}{c}\text { Baseline } \\
\mathbf{n = 2 1 4}\end{array}$ & $\begin{array}{c}\text { Endline } \\
\mathbf{n = 2 1 0}\end{array}$ & $\begin{array}{c}\text { Baseline } \\
\mathbf{n}=\mathbf{3 2 4}\end{array}$ & $\begin{array}{c}\text { Endline } \\
\mathbf{n}=\mathbf{3 1 0}\end{array}$ \\
\hline Ever used condom & 24 & 20 & 13 & 12 \\
\hline Used condom at last sex & $4(\mathrm{n}=181)$ & $6(\mathrm{n}=173)$ & $1(\mathrm{n}=246)$ & $6^{* *}(\mathrm{n}=227)$ \\
\hline Used condom in last month & 4 & $14^{* *}$ & 5 & $11^{* *}$ \\
\hline Using condom with FP method & $2(\mathrm{n}=179)$ & $5^{*}(\mathrm{n}=173)$ & $1(\mathrm{n}=245)$ & $6^{*}(\mathrm{n}=222)$ \\
\hline
\end{tabular}

* Significant at $p<0.05 ;{ }^{* *} P<0.01$

\section{Effect on offering and use of HIV testing}

The key outcome measures of this study were the effect of the interventions on providers offering FP clients an HIV test, and on clients' use of the HIV testing service. Table 12 shows that approximately two-thirds of new clients reported ever having an HIV test, and this did not change over time, nor was there any difference between the districts. This suggests during the duration of the study there were no external influences to increase testing among these women. For repeat clients, however, there were significant increases in the proportions reporting ever having had a test after the intervention was introduced (from $43 \%$ to $67 \%$ for the pooled sample), especially among those exposed to the testing model in Nyeri. (81\%). This suggests that exposure to the intervention, as indicated by the repeat clients at endline, has increased the likelihood of ever being tested and that the testing had been done as a result of the intervention.

Table 12: Clients' experience with HIV testing

\begin{tabular}{|l|c|c|c|c|c|c|c|c|}
\hline \multirow{2}{*}{} & \multicolumn{3}{|c|}{ Testing Model (Nyeri) } & \multicolumn{3}{c|}{ Referral Model (Thika) } \\
\cline { 2 - 10 } & Baseline & \multicolumn{2}{|c|}{ Endline } & \multicolumn{2}{|c|}{ Baseline } & \multicolumn{2}{|c|}{ Endline } \\
\cline { 2 - 10 } & $\%$ & $\mathbf{N}$ & $\%$ & $\mathbf{N}$ & $\%$ & $\mathbf{N}$ & $\%$ & $\mathbf{N}$ \\
\hline Ever had HIV test - new clients & 66 & 29 & 70 & 27 & 66 & 62 & 68 & 50 \\
\hline Ever had HIV test - repeat clients & 46 & 185 & $81^{* *}$ & 183 & 41 & 259 & $58^{* *}$ & 259 \\
\hline Client offered HIV test - new clients & 3 & 29 & $74^{* *}$ & 27 & 0 & 62 & $34^{* *}$ & 50 \\
\hline If offered, new clients accepted HIV test & 100 & 1 & 50 & 20 & 0 & & 65 & 17 \\
\hline Client offered HIV test - repeat clients & 1 & 185 & $56^{* *}$ & 183 & 0 & 259 & $27^{* *}$ & 259 \\
\hline If offered, repeat clients accepted HIV test & 50 & 2 & 61 & 103 & 0 & 1 & 72 & 69 \\
\hline Proportion of new clients being tested & 3 & 29 & $37^{* *}$ & 27 & 0 & 62 & $22^{* *}$ & 50 \\
\hline Proportion of repeat clients being tested & 1 & 185 & $34^{* *}$ & 183 & 0 & 259 & $19^{* *}$ & 259 \\
\hline Proportion of all clients being tested & 1 & 214 & $35^{* *}$ & 210 & 0 & 321 & $20^{* *}$ & 309 \\
\hline
\end{tabular}

* Significant at $p<0.05 ;{ }^{* *} \mathrm{P}<0.01$ 
One noticeable difference in Table 12 is that at baseline the proportions of new clients reporting ever having an HIV test were, on average, much higher (66\%) than among repeat clients (43\%). Anecdotal evidence from nurses at these facilities indicated that this may be because most of their new clients were women who had recently given birth (i.e. are postpartum clients) and the recent expansion of PMTCT services nationwide means that most pregnant women are now being tested. Indeed, 71 percent of new clients had a child younger than one year compared with only 45 percent of repeat clients, supporting these nurses' statements. At endline, the proportions of new clients (49\%) with babies under one year old was much lower than at baseline, which may explain why there was not been an increase in the proportion of new clients reporting HIV testing over time.

There were substantial and significant increases in the proportions of clients offered HIV testing during the consultation, from one percent at baseline to 39 percent (repeat) and 48 percent (new) at endline. Providers implementing the testing model were more likely than those implementing the referral model to offer their clients HIV testing, perhaps because they were able to offer the test themselves. For both models, the proportion of new clients being offered HIV testing (48\%) was higher than for repeat clients (39\%). The majority of repeat clients used injectables and pills, and so make regular visits to the clinic; consequently, the provider may know (or assume) that the client had been offered testing previously and so would not have made the offer during the consultation observed. It could also be due to the fact that FP counseling for repeat clients is always less thorough than for new clients, which is no different for integrated services.

Offering a client HIV testing is clearly an important action that providers should be encouraged to take, because $50-72$ percent of those offered testing accepted to have a test. Interestingly these proportions were higher among repeat clients, perhaps because the client had been offered the test several times before. Moreover, the proportions accepting a test were also higher in the referral model, which may substantiate the finding from client focus groups for a preference to be tested anonymously at another site.

Figure 3: Proportion of FP clients accepting to have an HIV test

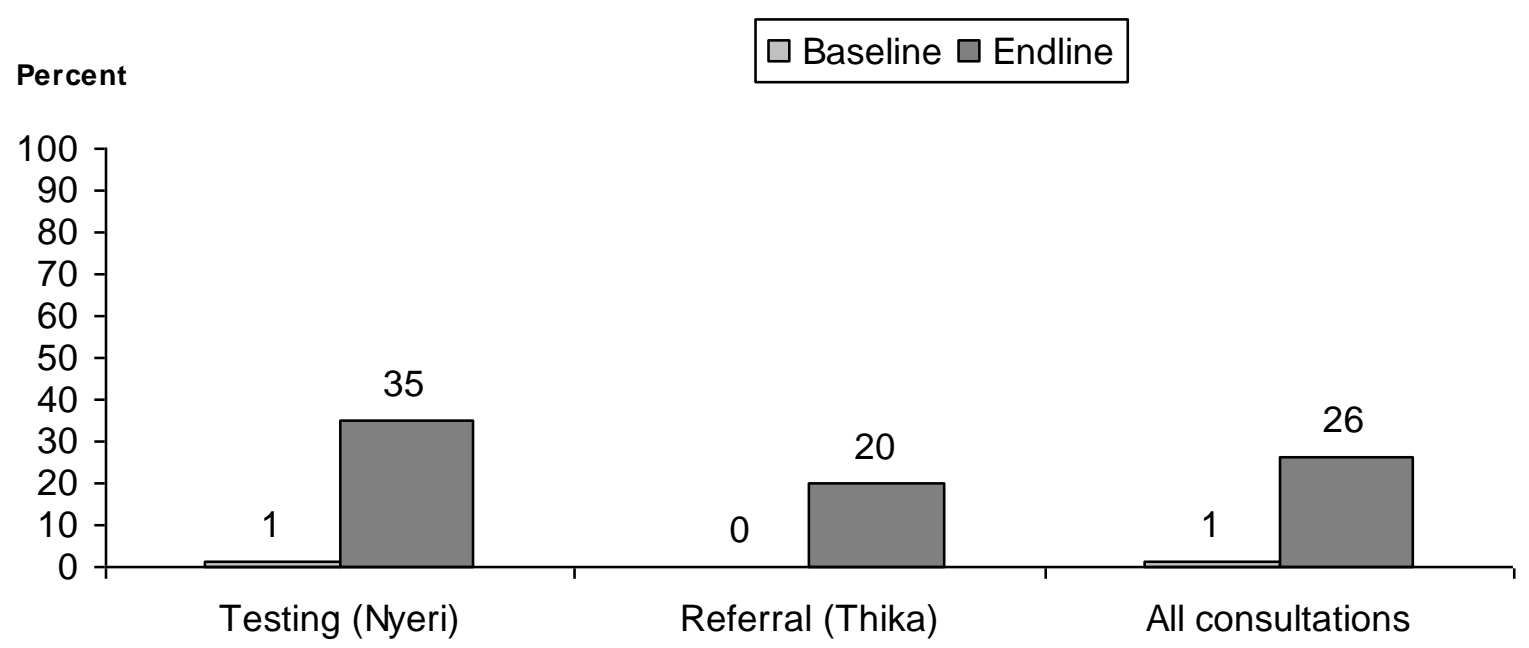


As shown on Figure 3, both interventions were highly effective in increasing the proportions of all FP clients actually having an HIV test after the FP consultation ${ }^{3}$. There were no differences between the proportions of new or repeat clients having an HIV test, despite the differences in being offered or not offered a test. Overall, approximately one-third of those choosing to have an HIV test (both new and repeat clients) had never had a test before; this proportion increased to one half for those attending referral model clinics, again probably because of the anonymity involved. These data clearly indicate that both models have the potential to substantially increase the number of women accessing and using HIV counseling and testing by offering the service during FP consultations.

\section{Cost analysis and incremental costs for scaling up}

Appendix 6 describes in detail the plan and findings from cost analysis. The cost analysis calculated the planning and preparation costs, most of which were incurred by this project, and the incremental costs for providing the VCT service in addition to the FP consultation. On average, the additional time required during the consultation to provide the VCT service was reasonable -3.03 minutes for the testing model (12.4 to 15.4 minutes) and 4.18 minutes in the referral model (13.2 to 17.4 minutes). Moreover, the additional costs per FP client were affordable, at $\$ 0.41$ for the testing model and $\$ 0.24$ for the referral model.

When deciding whether or not to scale up the intervention to other $\mathrm{MOH}$ facilities, the MOH will need to consider the resources required. For scaling up, we assume that much of the planning costs incurred during this OR study will not need to be repeated (development of job aids, development of training curriculum, and stakeholder meetings) and so are left out of the calculation. However, training of providers and the production of IEC materials will still be required, along with additional resources to support routine service delivery and supervision.

Table 13: Incremental cost per facility of scaling-up by model and level of care

\begin{tabular}{|c|l|c|c|c|c|c|}
\hline Model & $\begin{array}{l}\text { Level of } \\
\text { facility }\end{array}$ & $\begin{array}{c}\text { Financial } \\
\text { cost }^{4} \text { per } \\
\text { facility } \\
\text { (Ksh) }\end{array}$ & $\begin{array}{c}\text { Opportunity } \\
\text { cost }^{5} \\
\text { per facility } \\
\text { (Ksh) }\end{array}$ & $\begin{array}{c}\text { Total cost } \\
\text { per facility } \\
\text { (Ksh. \& \$) }\end{array}$ & $\begin{array}{c}\text { \# of } \\
\text { clients } \\
\text { tested per } \\
\text { facility }\end{array}$ & $\begin{array}{c}\text { Incremental } \\
\text { cost per client } \\
\text { tested } \\
\text { (Ksh \& \$) }\end{array}$ \\
\hline \multirow{3}{*}{ Testing } & Hospital & 388,834 & 46,430 & $435,264(\$ 6,218)$ & 1,110 & $392(\$ 5.60)$ \\
\cline { 2 - 7 } & Health Center & 277,133 & 19,212 & $296,345(\$ 4,234)$ & 460 & $645(\$ 9.21)$ \\
\cline { 2 - 7 } & Dispensary & 137,692 & 9,206 & $146,898(\$ 2,099)$ & 220 & $667(\$ 9.53)$ \\
\hline & & & & & & \\
\hline \multirow{3}{*}{ Referral } & Hospital & 194,695 & 66,012 & $260,707(\$ 3,724)$ & $\mathrm{n} / \mathrm{a}$ & $\mathrm{n} / \mathrm{a}$ \\
\cline { 2 - 7 } & Health Center & 130,953 & 20,634 & $151,587(\$ 2,166)$ & $\mathrm{n} / \mathrm{a}$ & $\mathrm{n} / \mathrm{a}$ \\
\cline { 2 - 7 } & Dispensary & 71,049 & 11,613 & $82,662(\$ 1,181)$ & $\mathrm{n} / \mathrm{a}$ & $\mathrm{n} / \mathrm{a}$ \\
\hline
\end{tabular}

3 It should be noted that for the referral model, it was not possible to confirm whether or not the woman actually had the test, but the fact that she requested it and was given a voucher to take to the VCT site suggests that these figures may be indicative of actual testing.

4 Financial cost is comprised of the resources used for the production of IEC materials, training of providers, additional supplies for service delivery (test kits and gloves), and additional supervision visits.

5 Opportunity cost is comprised of the value of the additional labour time required to provide integrated services to clients. If the $\mathrm{MOH}$ decides to hire additional providers, these costs would then become financial costs. 
For budgeting purposes, the $\mathrm{MOH}$ will be most interested in the cost of resources that will require additional financial expenditures as opposed to re-deployment of existing resources (e.g. staff time for service delivery). Therefore, we use the data presented in Appendix 6 to develop the following estimates of the cost of scaling-up the two models by type of facility (Table 13).

Introducing the testing model to a new facility is estimated to require resources worth $\$ 2,099$ to $\$ 6,218$ depending upon the level of facility. The proportions of total resources required are disaggregated as follows:

- Training providers requires between 47\% (hospital) and 69\% (dispensary);

- Service delivery requires between $34 \%$ (hospital) and 20\% (dispensary);

- Supervision visits require between 11\% (hospital) and 6\% (dispensary);

- IEC materials production requires between 9\% (hospital) and 4\% (health centre).

Based upon the experience of this study, the estimated incremental cost per FP client also counseled and tested for HIV in the testing model ranges from $\$ 5.60$ (hospital) to $\$ 9.53$ (dispensary), which compares well with the estimated per client cost for stand-alone VCT of \$27 in Kenya (Sweat and Coates 2000).

Introducing the referral model to a new facility is estimated to require resources worth $\$ 1,181$ to $\$ 3,724$ depending upon the level of facility. As testing is not done within the facility, or at least within the FP clinic, there are no additional costs incurred for HIV testing. The proportions of total resources required are disaggregated as follows:

Training providers requires between 40\% (hospital) and 69\% (health centre);

Service delivery requires between $25 \%$ (hospital) and 14\% (health centre or dispensary); Supervision visits require between $18 \%$ (hospital) and 10\% (health centre or dispensary); IEC materials production requires between $17 \%$ (hospital) and $8 \%$ (health centre).

\section{STUDY LIMITATIONS}

The study had the following limitations. Firstly, the number and profiles of participating health facilities were different in each model. Thika had two hospitals while Nyeri had only one and Thika had eight health centers compared with four in Nyeri. Second, national HIV surveillance data shows that HIV prevalence is estimated at 10 percent in Thika compared with 2.4 percent in Nyeri, which may influence client awareness, behaviors and attitude towards HIV testing in each district. Third, Thika is primarily an urban and peri-urban district while Nyeri District is largely rural, which influences the mobility of health facility clients.

\section{CONCLUSIONS}

The study demonstrated that both models were feasible and acceptable to providers and to clients as means of integrating and linking HIV prevention counseling, condom promotion and counseling and testing with FP services, and is effective in increasing quality of care and service utilization. Specific findings showed that: 
Integrating counseling and testing for HIV into FP services is feasible and acceptable: The majority of facilities had the capacity to integrate HIV prevention counseling and provision of or referral for CT services within existing FP services. Provision of both services jointly was perceived by clients to be beneficial and attractive and was welcomed by providers as an opportunity to provide a comprehensive service that better meets their clients' needs. Existing differences between clinics in each district meant that some were better prepared than others to offer integrated services.

Quality of family planning counseling improved: Significant improvements were observed in the quality of FP counseling and in the rapport established with clients in both models. Discussion of reproductive intentions remained a weakness however.

Quality of counseling on STI/HIV issues improved: As was anticipated, levels of counseling on STI/HIV issues were low at baseline. After introduction of the BCS Plus approach, there were marked increases in discussions about STIs and HIV/AIDS generally and about the risk factors, but discussions of the client's personal sexual history and behaviors remained low, and need so needs more attention.

Counseling on condoms and dual protection and their use improved: Condoms were rarely mentioned or discussed at the baseline, especially in Thika district. Training and use of the BCS Plus significantly increased discussions around condoms and their use. Reported use of condoms at last sex, in last month, and with another contraceptive method all increased significantly.

Counseling on HIV CT increased during FP consultations: One of the focus items of the intervention was to increase counseling on HIV counseling and testing during the FP consultations. At baseline, the proportion of consultations in which VCT is mentioned was quite high at 39 percent overall. Introduction of the intervention led, however, to substantial increases, with HIV counseling and testing being mentioned in 88 percent of all consultations at endline. Discussions of the client's own serostatus increased dramatically also, from an average of 24 to 81 percent of consultations.

Use of the BCS Plus approach significantly improved the quality of integrated services: Summary scores were calculated for 27 counseling items that providers were trained to cover when providing integrated services. The mean summary scores for those consultations when the BCS Plus approach was used were much higher (18.86) than for consultations when the BCS Plus was not used (12.19), clearly indicating that the BCS Plus approach is an effective means for improving the quality of counseling when providing integrated services. The approach was particularly effective in Thika district, where quality of care scores almost doubled when it was used.

Clients' offered and obtaining HIV CT increased: Approximately two-thirds of all new clients had ever had an HIV test, and this did not change over time, nor was there any difference between the districts, suggesting that there have been no external influences on testing among women not exposed to the intervention. For repeat clients, there were significant increases in the proportions ever having a test after the intervention (from $43 \%$ to $67 \%$ ), and especially among those exposed to the testing model. There were substantial increases in the proportions of both new and repeat clients offered HIV testing during the consultation - from $0-3$ percent at 
baseline to $27-74$ percent at endline. Providers implementing the testing model were much more likely than those implementing the referral model to offer HIV testing, which may be because they were able to offer the test themselves. For both models, the proportions of new clients being offered HIV testing $(34 \%-74 \%)$ were higher than for repeat clients $(27 \%-56 \%)$, which may be due to the majority of repeat clients using injectables and so the provider may have assumed that the client had been offered testing previously. Overall, $50-72$ percent of FP clients offered testing accepted to have a test, and these proportions were higher among repeat clients and for the referral model, which may be due to a preference for anonymity at another site. The proportions of all FP clients actually having an HIV test after the FP consultation were 20 percent in the referral model and 35 percent in the testing model, with little difference between new or repeat clients. These data clearly indicate that both models are effective in substantially increasing the number of women accessing HIV counseling and testing through offering the service during FP consultations.

Incremental costs for integrating CT into FP services are affordable: On average, the additional time required during an FP consultation to provide the CT service was reasonable 3.03 minutes for the testing model (from 12.4 to 15.4 minutes) and 4.18 minutes in the referral model (from 13.2 to 17.4 minutes), and the additional costs per FP client for this time were affordable, at $\$ 0.41$ for the testing model and $\$ 0.24$ for the referral model. For planning scalingup of the testing model, the estimated incremental cost per FP client who is also counseled and tested for HIV ranges from $\$ 5.60$ (hospital) to $\$ 9.53$ (dispensary). This compares favorably with an estimated cost per client at stand-alone VCT sites of $\$ 27$.

Drawing from the lessons learned, the key programmatic recommendations for institutionalizing and scaling up this approach are:

- Ensuring that provincial and district level MOH managers and trainers, from both reproductive health and STI/HIV control, lead activities to build capacity and introduce the new service delivery protocols is critical.

- District and facility action plans can be used as management tools for ensuring resource mobilization and allocation and securing commitment to providing integrated services.

- The Balanced Counseling Strategy Plus approach and tools has enhanced the range of services offered to FP clients and significantly improved the quality of care.

- On-the-job updates by district level managers and trainers rapidly expanded the number of health providers able to provide HIV CT for FP clients, but for sustainability the existing FP service delivery protocols need to be reviewed and revised to mainstream this approach in high STI/HIV prevalence settings.

- Continuous availability of both FP and HIV CT commodities was a key factor in ensuring an attractive and accessible service.

- Both models are effective in increasing the number of women testing for HIV, both for the first time and for a repeat test.

- Clinics that can offer HIV testing on-site should also consider offering clients the option of being referred elsewhere for the test, given the evidence suggesting that anonymity is important for these FP clients who may meet the same provider on several occasions. 


\section{UTILIZATION OF RESEARCH FINDINGS}

The preliminary findings and lessons learned were communicated to key stakeholders in August 2007. Drawing on this evidence, the DRH and NASCOP recommended that integration of HIV CT within FP services and especially using the testing model where possible, should be adopted, institutionalized and scaled up nationwide. A follow-up meeting between the $\mathrm{MOH}$ and FRONTIERS in September 2007 planned for national and provincial-level training of trainers (TOTs). By end of October 2007, about 40 provincial-level TOTs from the remaining seven provinces had been trained by NASCOP and DRH, in collaboration with FRONTIERS and with funding from PEPFAR through the US Centers for Disease Control (CDC), to build provinciallevel capacity for training district-level staff. By mid-July 2008, the USAID-funded APHIA II partners had supported the Provincial Health Management Teams to train 93 providers in Central province and 33 providers in Nairobi province. By the end of 2008, Nairobi province plans to train a further 90 providers, Eastern Province will train a further 20 TOTs and 200 providers, and Coast Province will train 120 providers. Lessons from this study have been presented at several national and international workshops and conferences. 


\section{REFERENCES}

Boateng J., et al. 2006. Costs of Reproductive Health Services Provided by Four CHAG Hospitals, FRONTIERS Final Report: Population Council, Washington DC

Bradley H. et al. .2007. "HIV and Family Planning Service integration and Voluntary HIV counseling and testing client composition in Ethiopia", Presentation at the Annual Meeting of the Population Association of America: New York, USA.

Bruce, J. 1990. "Fundamental Elements of Quality of Care: A Simple Framework," Studies in Family Planning 21, no. 2: 61-91

Creek T., et al. 2007. "Successful Introduction of Routine Opt-Out HIV Testing in Antenatal Care in Botswana", Acquir Immune Defic Syndr, Vol 45, No. 1.

Central Bureau of Statistics, Ministry of Health, and ORC Macro. 2004. Kenya Demographic and Health Survey 2003. Calverton, Maryland: CBS, MOH, and ORC Macro.

Leon, F. et al. 2003. Testing Balanced Counseling to Improve Provider-Client Interaction in Guatemala's MOH Clinics, FRONTIERS Final Report: Population Council, Washington $\mathrm{DC}$

MOH. 2005. Draft Strategy for the Integration of HIV Voluntary Counseling and Testing (VCT) Services and Family Planning Services, MOH: Nairobi, Kenya.

MOH. 2005. National PMTCT Trainers Manual NASCOP/NLTP: Nairobi, Kenya.

MOH. 2005. Sexually Transmitted and Other Reproductive Tract Infections: A Guide to Essential Practice, Division of Reproductive Health: Nairobi, Kenya.

MOH 2007. Costing Post Rape Care Services in the public health sector in Kenya, Division of Reproductive Health: Nairobi, Kenya.

Homan, R. et al. 2006. "Cost of introducing two different models of integrating VCT for HIV within family planning (FP) clinics in South Africa" Paper presented at the International Meeting on Integrating HIV and RH, $9^{\text {th }}-10^{\text {th }}$ October, Addis Ababa, Ethiopia.

Reynolds, H. et al. 2006. Integrating Family Planning Services into Voluntary Counseling and Testing Centers in Kenya: Operations Research Results, Family Health International: Nairobi, Kenya.

Sweat M and T. Coates. 2000. "Reducing HIV-1 in Kenya and Tanzania", The Lancet, Vol. 356, Issue 9241: 1602-1603

WHO (2002): The Safety and Feasibility of Female Condom Reuse. Report of a WHO Consultation 28-29 January 2002, Geneva. 


\section{Appendix 1: Algorithm for the Balanced Counseling Strategy Plus}

\section{Pre-Choice}

Establish and maintain a warm, cordial relationship. Briefly mention the range of FP, RTI/STI/HIV services offered in the FP clinic or through referral. Assure the client that her/his FP needs will be addressed. Emphasize to the client that during the consultation, any other RH needs such as RTIs/STIs and HIV will also be addressed depending on her/his individual circumstance. Listen to the client's contraceptive needs.

Rule out pregnancy using the method card with the checklist of questions.

\begin{tabular}{|l|l|}
\hline If client answers & Then \\
\hline "Yes" to any of the questions & Continue to Step 3. \\
\hline \multirow{3}{*}{ "No" to all of the questions } & $\begin{array}{l}\text { Send for a pregnancy test or refer to prenatal clinic. } \\
\text { Ask client to return when she has her menstruation. } \\
\text { Provide a back-up method, such as condoms. } \\
\text { Discuss with client sub steps 11 to 15 below on RTI/STI/HIV } \\
\text { prevention, risk assessment and C\&T. }\end{array}$ \\
\hline
\end{tabular}

Display all of the method cards. If the client wants a particular method, go to Step 7.

Ask all of the following questions and set aside method cards based on the client's response:

Do you wish to have children in the future?

If "Yes", set aside vasectomy and tubal ligation cards and explain why.

If "No", keep all cards and continue.

Are you breastfeeding an infant less than 6 months old?

If "Yes", set aside the combined oral contraceptives (pills) card and explain why. Discuss the option of the mini pill.

If "No", set aside the LAM card and explain why.

Does your partner support you in family planning?

If "Yes", continue.

If "No", set aside the natural methods (Billings, Rhythm, Standard Days) and barrier methods (condoms, vaginal tablets) cards and explain why.

Are there any methods that you do not want to use or have not tolerated in the past?

If "Yes", set aside the cards the client does not want and explain why.

If "No", keep the rest of the cards.

\section{(b) Method Choice}

Give information on the methods that have not been set aside, in order of their efficacy.

Arrange the remaining cards in order of effectiveness (number on back side of each card).

In order of efficacy (lowest no. to highest), read the 4 attributes of each method not set aside.

Ask the client to choose the method that is most convenient for her/him.

Determine if there are any limitations for the method chosen, using the brochure.

Together with the client, review the brochure of the method chosen and its contraindications.

If the method is contraindicated, ask the client to select another method from the cards that remained repeating the process from Step 5.

(c) Post-Choice

Inform the client about the method, using the brochure of the method as a counseling tool (Describe action mechanism, give use instructions, side effects and alarm signs).

Determine client's comprehension and reinforce key information if needed.

Make sure the client has made a definite decision and give her/him the method chosen and/or a referral and back-up method, depending on the client's needs.

Encourage the client to involve partner(s) in contraception either through discussion or to come to the clinic

\section{2. (d) RTI/STI/HIV Prevention, Risk Assessment and Counseling and Testing}

Discuss RTI/STI/HIV transmission and prevention.

Conduct HIV/AIDS Risk assessment. If RTI/STI symptoms, treat syndromically.

Discuss dual protection. Offer condoms and instruct correct and consistent use. Ask if client knows how to use a condom. Demonstrate use if required. If client knows let her/him do a return demonstration.

Discuss and offer client opportunities for Counseling and Testing for HIV. If willing, test and counsel client for negative or positive test results as per the National Protocol.

Give follow-up instructions, condom pamphlet and pamphlet of the method chosen.

Encourage the client to involve partner(s) in HIV testing either through discussion or to come to the clinic

Complete the counseling session by giving the client a follow-up appointment; invite the client to return if s/he has problem and end the session with a warm and cordial attitude. 


\section{Appendix 2: Example of BCS-plus counseling cards}

VCT Awareness (front side)

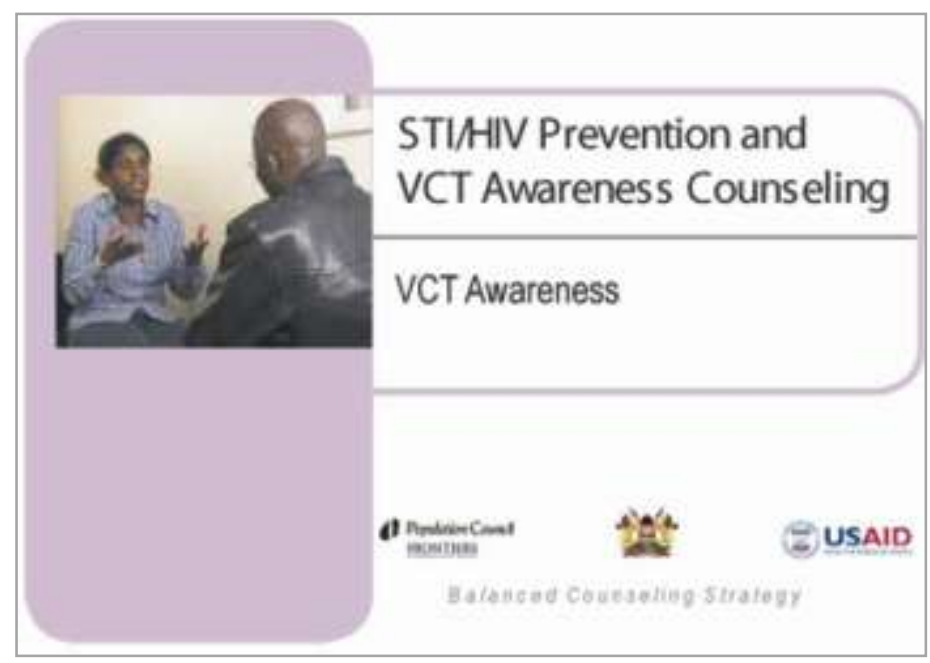

\section{VCT Awareness (back side)}

\section{STI/HIV Prevention and VCT Awareness Counseling}

- A test is available to determine whether or not a person is infected with HIV. The test involves taking a sample of blood.

- When a person is infected it can take three-six months for the body to produce levels of the antibodies that can be detected by the test

- Knowing your status can help you make decisions about protecting yourself and your sexual partners, having children, etc.

- No one can force you to have the test. Taking an HIV test is voluntary

- Results are confidential

- Positive results mean you are infected and can transmit the virus to others

- Negative results can mean you are not infected or that you are in the window period. Retest in three months. If still negative, it does not mean that you cannot get HIV at a later stage. Retest in future if you have had unprotected sex

- HIV is an STI. It is advisable to ask your sexual partners to be tested

- The test is free and available at clinics, hospitals, and other places

\section{Dual protection (front side)}

\section{Dual protection (front side)}

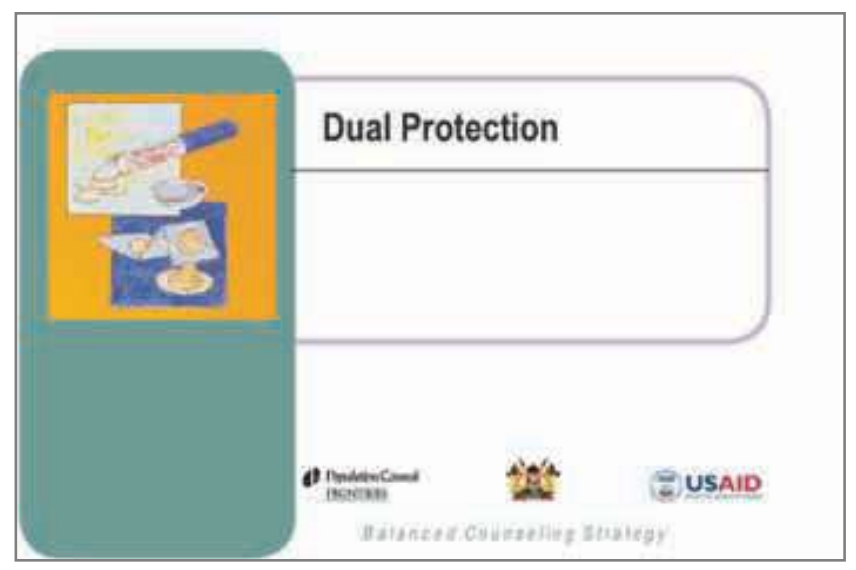

\section{Dual protection (back side)}

\section{Dual Protection}

- Protects against both STI and HIV and prevents unwanted pregnancies

- Abstinence from intercourse/all types of penetrative sex

- Using contraception correctly and consistently in a mutually faithful relationship with an uninfected partner

- Using correctly and at each intercourse the female or male condom

- Using your chosen method and a male or female condom at the same time

- Show how to correctly use condoms

- Give information on where to get condoms 


\section{Appendix 3: Example of BCS-plus brochure \\ Combined oral contraception - front side}

\begin{tabular}{|c|c|}
\hline \multicolumn{2}{|c|}{ Follow these guidelines if you miss 1 of more pllts: } \\
\hline If yout & Dothis: \\
\hline $\begin{array}{l}\text { - Missed } 1 \text { or } 2 \text { pills } \\
\text { - OR - } \\
\text { - } 5 \text { tarted a new packet } 1 \text { or } \\
2 \text { days late }\end{array}$ & $\begin{array}{l}\text { - Take a pill as scon as possible, } \\
\text { - Keep taking pills as us ual. (You may end up taking } \\
2 \text { pills at the same time or on the same day.) }\end{array}$ \\
\hline 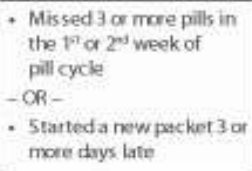 & $\begin{array}{l}\text { - Take a pill as scon as pos sible, } \\
\text { - Use a tackup rnethod (condonr) or awcid sex for } \\
\text { the next } 7 \text { drys. } \\
\text { - If you had unprotected sex in the past } 5 \text { days, } \\
\text { consider taking emergency contraceptive pills } \\
\text { (ECPs). }\end{array}$ \\
\hline $\begin{array}{l}\text { Missed } 3 \text { or more pills in the } \\
3^{6} \text { week of the pill cycle }\end{array}$ & $\begin{array}{l}\text { - Take a pill as scon as pos sible, } \\
\text { - Firish all hormanal pills in the pariogt. For a } 28 \text {-pill } \\
\text { packet, thow away the } 7 \text { non-hormornal pills. } \\
\text { - Start a new packet the next day. } \\
\text { - Use a backup method (condorrs) or avoid sex for } \\
\text { the next } 7 \text { days. } \\
\text { - If you had unpectected sex in the past } 5 \text { chys, } \\
\text { consider takingECPs. }\end{array}$ \\
\hline $\begin{array}{l}\text { Missed any non-hormoral } \\
\text { pills (last } 7 \text { pills in 28-day pill } \\
\text { pecket) }\end{array}$ & $\begin{array}{l}\text { - Discard the mis sed non-hormonsl pillis). } \\
\text { - Keep taking the pill, one each day. Start the new } \\
\text { packetas us tal. }\end{array}$ \\
\hline
\end{tabular}

Return to the health care facility anytime if:

- You have any questions or problems.

- You have missed 3 or more pllls, had sex in the past 5 -days, and want to avold pregrancy. You can take ECPs.

- You develop ary health peoblerro.

- Ycu think you rray be pregrant.

- After 1 yeser for follow-up and to get more pills.

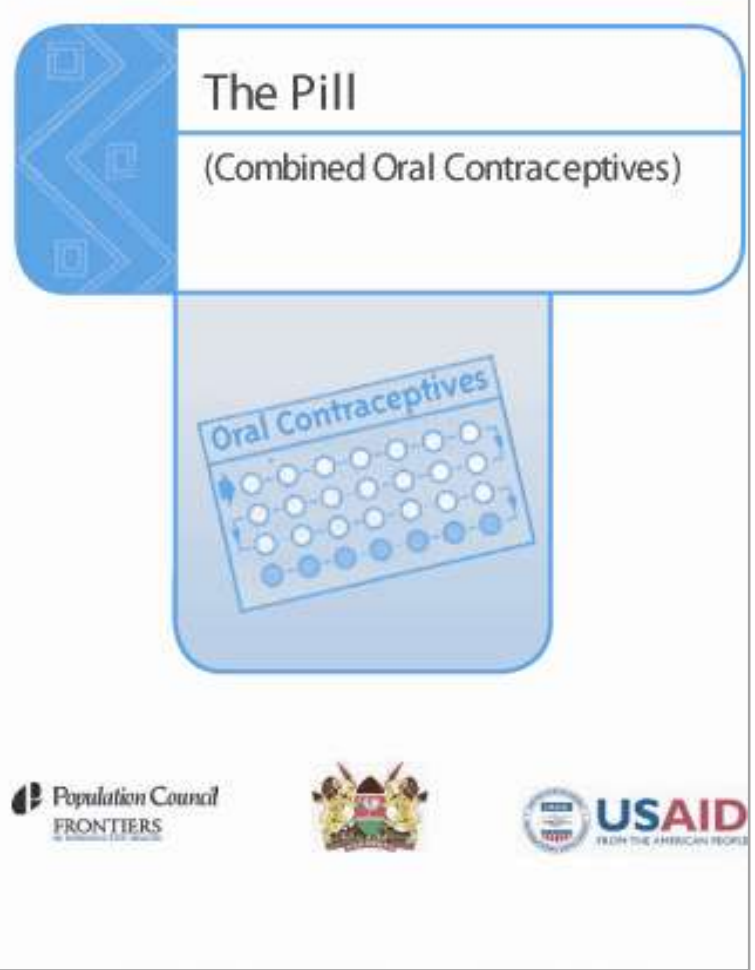

\section{Combined oral contraception - inside side}

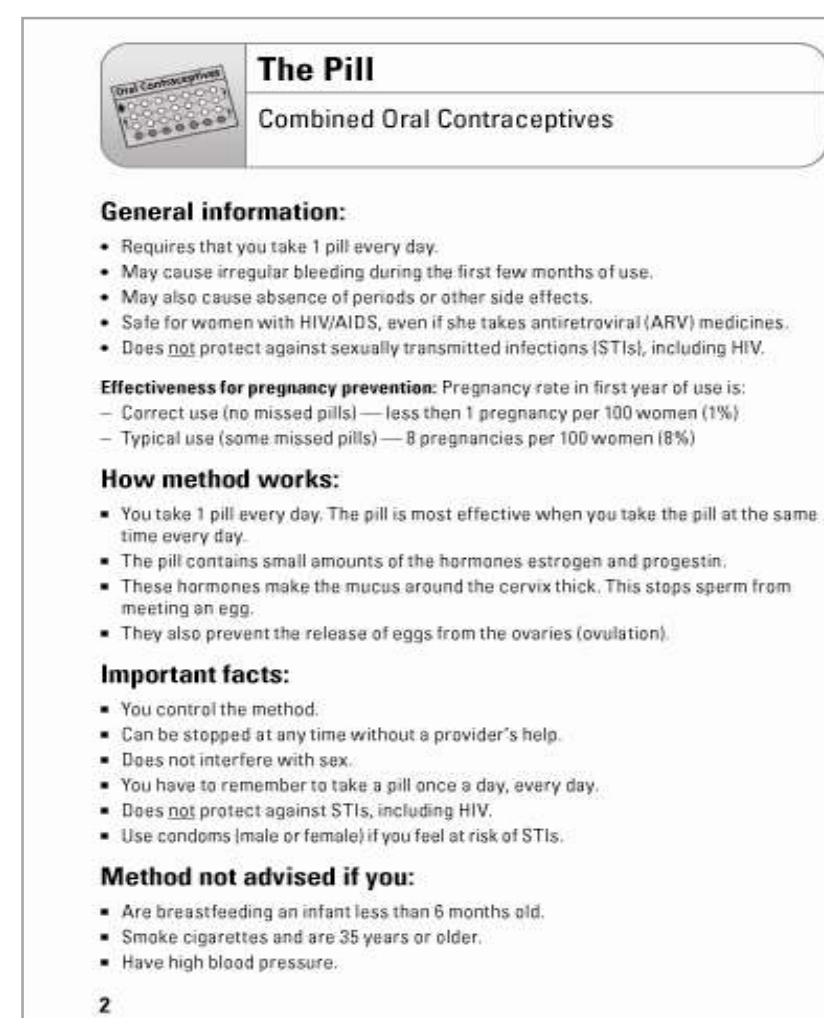

- Have certain uncommon serious diseases of the heart, blood vessels or liver, 0 breast cancer. Discuss with your provider

- Have gall bladder disease, Discuss with your provider.

- Have migraine headaches la type of severe headachel and are 35 years or older

- Have migraine aura (sometimes seeing a growing bright spot in one eyel at any age.

- Take medicine for seizures or take rifampicin

\section{Side effects:}

- Lighter periods, irregular bleeding, or spotting between menstrual periods. These are common in the first 3 months of use.

- May also cause infrequent bleeding or absence of menstrual periods (amenorrhea).

- May cause headaches, breast tenderness, or weight change. These usually lessen or stop within the first few months.

- These are normal and not signs of illness

- Not allwamen have these side effects

\section{Health benefits:}

- Helps prevent against cancer of the uterus and ovaries.

- Helps prevent pelvic inflammatory disease (PID)

- May help protect against ovarian cysts and anemia.

- Reduces menstrual cramps and menstrual bleeding problems.

How to use:

- Begin with the first pill in the packet as directed by your provider.

- TAKE1 PILL EVERY DAY until you finish the packet.

- Take the pill at the same time every day, for example every night before going to bed.

- If you vomit within 2 hours of taking a pill, take another pill from your packet as soon as possible. Continue taking pills as usual.

- If vomiting or diarrhea continues for more than 2 days, follow instructions for $1-2$

missed pills on the next page.

- Have a new packet of pills on hand before you finish your 28-day or 21-day packet

\begin{tabular}{|l|l|}
\hline If using: & Do this: \\
\hline A 28-day packet and you finish the packet & Begin a new packet immediately. \\
\hline A 21-day packet and you finish the packet & $\begin{array}{l}\text { Wait no more than 7 days before } \\
\text { starting the new packet. }\end{array}$ \\
\hline
\end{tabular}

- If you get your pills from a pharmacy, use the instructions in this brochure.

- If you miss any pills, follow the instructions on the next page 


\section{Appendix 4: Study facilities and client load}

\section{Thika District}

\begin{tabular}{cccc} 
Selected health facility & Division & 2005 FP load & 2005 VCT load \\
\hline Hospitals & Municipality & 9,363 & 2,483 \\
Thika District & Gatundu & 6,791 & 987 \\
Gatundu SD & Ruiru & 3,660 & 1,375 \\
Health Centers & Kamwangi & 3,084 & 716 \\
Ruiru & Gatanga & 2,520 & 189 \\
Igegania & Kamwangi & 2,328 & 322 \\
Gatura & Thika Rural & 1,716 & 116 \\
Ngorongo & Gatundu South & 1,417 & 200 \\
Munyu & Gatanga & 1,344 & 281 \\
Ngenda & Thika Rural & 1,296 & 193 \\
Kirwara & & \\
Ngoliba & Kakuzi & 2,712 & - \\
Dispensaries & Gatundu & 1,860 & - \\
Ithanga & Gatanga & 1,656 & - \\
Gitare & Kamwangi & 1,596 & - \\
Mukurwe & & & \\
Gakoe & \multicolumn{3}{c}{}
\end{tabular}

\section{Nyeri District}

\begin{tabular}{ccc} 
Selected health facility & Division & 2005 FP load \\
\hline $\begin{array}{c}\text { Hospitals } \\
\text { Provincial General } \\
\text { Health Centers }\end{array}$ & Municipality & 6919 \\
Gichira & Tetu & 1464 \\
Ngorano & Mathira & 4238 \\
Warazo & Kieni West & 1624 \\
Belle View & Kieni West & 1977 \\
Dispensaries & & \\
Kiaguthu & Othaya & 1211 \\
Mweru & Mukurweini & 1104 \\
Kiganjo & Municipality Division & 1600 \\
Island & Kieni East & 1376 \\
\hline
\end{tabular}


Appendix 5: Availability of equipment, supplies and FP and STI/HIV services

\begin{tabular}{|c|c|c|c|}
\hline \multirow[t]{2}{*}{ Percentage of facilities with: } & $\begin{array}{l}\text { Pooled } \\
\text { Sample }\end{array}$ & $\begin{array}{l}\text { Testing } \\
\text { Model }\end{array}$ & $\begin{array}{c}\text { Referral } \\
\text { Model }\end{array}$ \\
\hline & $(\mathrm{N}=23) \%$ & $(\mathrm{~N}=9) \%$ & $(\mathrm{~N}=14) \%$ \\
\hline BP Machine & 100 & 100 & 100 \\
\hline Stethoscope & 96 & 100 & 93 \\
\hline Weighing machine & 96 & 89 & 100 \\
\hline Examination light & 38 & 25 & 46 \\
\hline Forceps & 100 & 100 & 100 \\
\hline Scissors & 100 & 100 & 100 \\
\hline Suction machine & 52 & 63 & 46 \\
\hline Large tray or kidney dish & 100 & 100 & 100 \\
\hline Green towels & 68 & 88 & 57 \\
\hline Examination couch & 100 & 100 & 100 \\
\hline Screen for privacy & 83 & 78 & 86 \\
\hline Display board for FP methods & 43 & 75 & 23 \\
\hline Penile model & 74 & 56 & 86 \\
\hline Portable light & 43 & 44 & 43 \\
\hline Sterilization set/autoclave & 59 & 63 & 57 \\
\hline Autoclaving tape & 55 & 50 & 57 \\
\hline Sterilizing drums & 57 & 56 & 57 \\
\hline Total & 74 & 76 & 74 \\
\hline \multicolumn{4}{|c|}{ Availability of FP Supplies } \\
\hline IUCD kit & 74 & 83 & 69 \\
\hline Disposable towels & 14 & 25 & 7 \\
\hline Green towels & 65 & 78 & 57 \\
\hline Total & 46 & 52 & 43 \\
\hline \multicolumn{4}{|c|}{ Availability of HIV/STI Supplies } \\
\hline Syringes and needles & 90 & 100 & 85 \\
\hline Laboratory reagents for urine & 100 & 100 & 100 \\
\hline Laboratory reagents for HIV & 44 & 63 & 30 \\
\hline Laboratory reagents for HB & 74 & 75 & 73 \\
\hline Laboratory reagents for VDRL & 95 & 88 & 100 \\
\hline Clean gloves & 100 & 100 & 100 \\
\hline Sterile gloves & 68 & 88 & 57 \\
\hline Gauze & 100 & 100 & 100 \\
\hline Cotton wool & 100 & 100 & 100 \\
\hline Hand washing soap & 91 & 100 & 86 \\
\hline Rapid test kits & 65 & 56 & 71 \\
\hline IEC materials for VCT & 45 & 22 & 62 \\
\hline
\end{tabular}




\begin{tabular}{|c|c|c|c|}
\hline Percentage of facilities with: & $\begin{array}{l}\text { Pooled } \\
\text { Sample }\end{array}$ & $\begin{array}{l}\text { Testing } \\
\text { Model }\end{array}$ & $\begin{array}{c}\text { Referral } \\
\text { Model }\end{array}$ \\
\hline Total & 81 & 83 & 80 \\
\hline \multicolumn{4}{|c|}{ Availability of Basic Infrastructure } \\
\hline Room for privacy & 95 & 100 & 92 \\
\hline Clean water & 96 & 100 & 93 \\
\hline Power to ensure fridge remains functional $24 \mathrm{hrs} /$ day & 95 & 88 & 100 \\
\hline Functional fridge & 100 & 100 & 100 \\
\hline $\mathrm{MCH} / \mathrm{FP}$ waiting area is shaded and with seats & 95 & 100 & 93 \\
\hline Furniture & 91 & 100 & 85 \\
\hline Clients have access to toilets & 95 & 100 & 92 \\
\hline Incinerator & 44 & 33 & 50 \\
\hline Placenta pit & 86 & 88 & 85 \\
\hline Total & 84 & 83 & 84 \\
\hline \multicolumn{4}{|c|}{ Provision of FP Services } \\
\hline Combined oral contraceptives pills (COC) & 95 & 89 & 100 \\
\hline Progestin-only contraceptive pills (POP) & 76 & 89 & 67 \\
\hline Emergency contraceptive pill (ECP) & 61 & 86 & 45 \\
\hline Progestin only injectable contraceptive - DMPA & 100 & 100 & 100 \\
\hline Progestin only contraceptive implants - Norplant & 21 & 13 & 27 \\
\hline Progestin only contraceptive implants - Jadelle & 30 & 25 & 33 \\
\hline Intrauterine Contraceptive Device (IUCD) & 85 & 100 & 73 \\
\hline Voluntary surgical contraception & 21 & 33 & 13 \\
\hline Male condom & 96 & 100 & 93 \\
\hline Female condom & 86 & 100 & 75 \\
\hline Lactational amenorrhea method (LAM) & 67 & 63 & 70 \\
\hline Natural family planning & 58 & 67 & 50 \\
\hline Counseling for FP & 100 & 100 & 100 \\
\hline IEC materials for FP & 84 & 100 & 73 \\
\hline Referral & 89 & 100 & 82 \\
\hline Total & 63 & 73 & 56 \\
\hline \multicolumn{4}{|c|}{ Provision of HIV/STI Services } \\
\hline HIV General counseling & 95 & 100 & 91 \\
\hline Counseling for HIV/AIDS within FP services & 61 & 75 & 50 \\
\hline HIV IEC & 80 & 89 & 73 \\
\hline HIV Behavior change communication & 95 & 100 & 92 \\
\hline Routine testing for HIV in STI \& TB clinics & 32 & 38 & 27 \\
\hline Routine testing for HIV in ANC PPC clinics & 65 & 75 & 58 \\
\hline HIV Pre-test counseling & 80 & 88 & 75 \\
\hline HIV Post-test counseling & 70 & 63 & 75 \\
\hline Provision of ARVs in PMTCT/CCC & 60 & 63 & 58 \\
\hline
\end{tabular}




\begin{tabular}{|c|c|c|c|}
\hline Percentage of facilities with: & $\begin{array}{l}\text { Pooled } \\
\text { Sample }\end{array}$ & $\begin{array}{l}\text { Testing } \\
\text { Model }\end{array}$ & $\begin{array}{c}\text { Referral } \\
\text { Model }\end{array}$ \\
\hline Provision of PMTCT services & 76 & 75 & 77 \\
\hline Counseling for dual protection from HIV & 68 & 75 & 64 \\
\hline Gender-based violence recovery services, PEP & 26 & 14 & 33 \\
\hline STI Treatment & 100 & 100 & 100 \\
\hline STI Counseling & 100 & 100 & 100 \\
\hline Partner follow-ups for STIs & 100 & 100 & 100 \\
\hline STI Information, education and communication (IEC) & 91 & 89 & 93 \\
\hline Laboratory diagnosis of STI & 86 & 86 & 86 \\
\hline Provision of antibiotics/appropriate drugs for STIs & 100 & 100 & 100 \\
\hline Syndromic management of STIs & 100 & 100 & 100 \\
\hline Dual protection from STIs & 100 & 100 & 100 \\
\hline Total & 73 & 77 & 71 \\
\hline \multicolumn{4}{|l|}{$\begin{array}{ll} & \text { Staffing } \\
\end{array}$} \\
\hline $\begin{array}{l}\text { Average number of enrolled nurses/midwives in } \\
\mathrm{MCH} / \mathrm{FP} \text { health facilities }\end{array}$ & 6 & 4 & 7 \\
\hline $\begin{array}{l}\text { Average number of registered nurses/midwives in } \\
\mathrm{MCH} / \mathrm{FP} \text { units in health facilities }\end{array}$ & 1 & 1 & 1 \\
\hline
\end{tabular}




\section{Appendix 6: Measuring and analyzing incremental costs}

In the interventions tested, clients seeking PF information and services also receive information and services on HIV, including counseling and testing. In addition, some of the clients receive services pertaining to RTIs/STIs and referral to other facilities in situations where a particular service may not be available. The costing framework for integrating counseling and testing for HIV into FP services takes into account only the additional costs incurred for integrating these services. From the figure below, these costs are represented by the interface between FP and HIV/AIDS services, as well as other costs shown in the intervention arm.

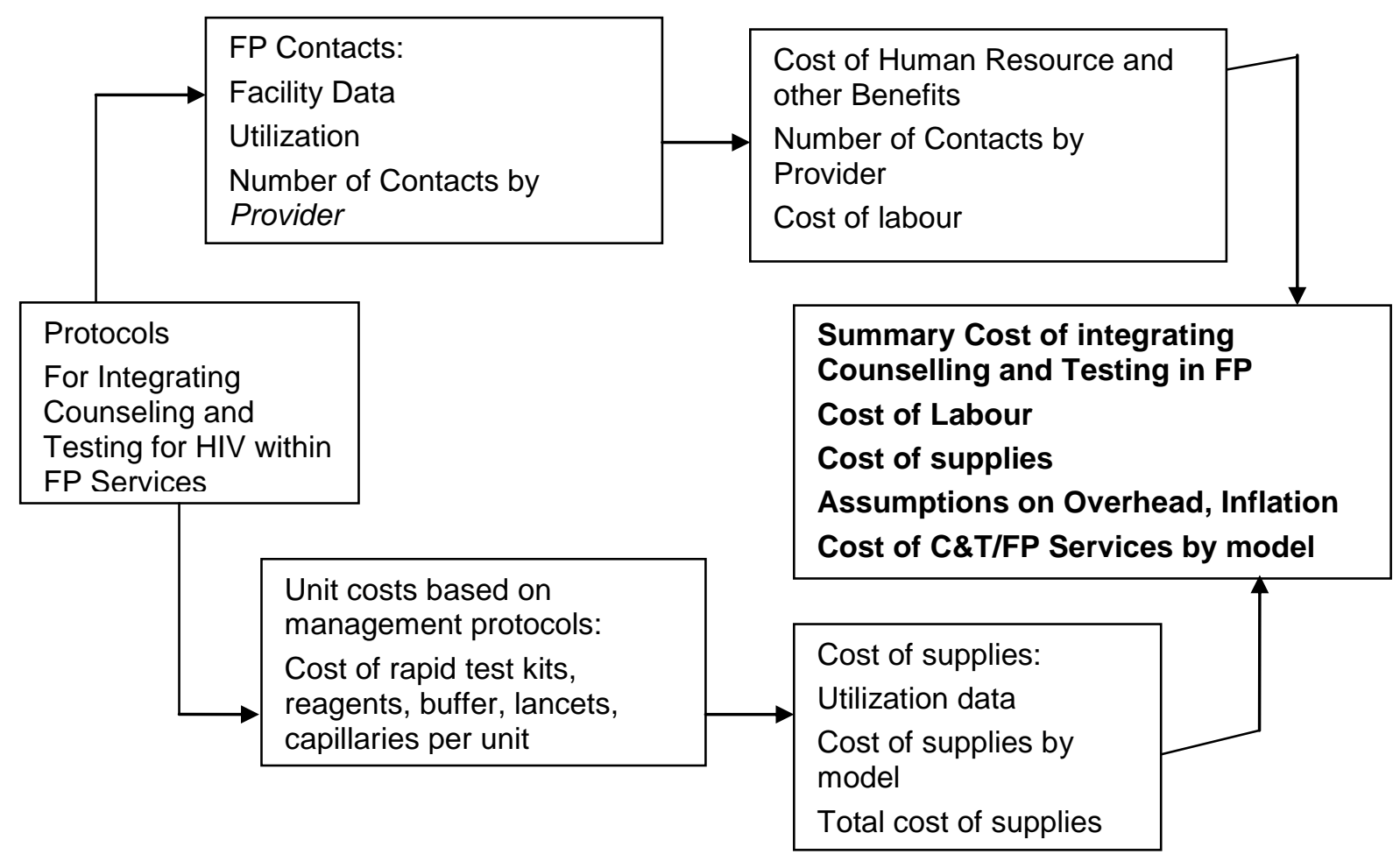

\section{Cost Estimation}

The purpose of this cost estimate is to provide guidance to the $\mathrm{MOH}$ as to the additional resources required to introduce C\&T for HIV into facility-based FP services. The estimation of incremental costs used a four-step process:

1. Identification of all resources used to introduce VCT services.

2. Measuring resources in their natural units (quantification) e.g. number of HIV test kits supplied.

3. Converting natural units into cost estimates (i.e. quantity $\mathrm{x}$ unit price).

4. Estimating incremental costs associated with introducing each of the models.

Data were collected retrospectively (before introduction of the interventions) and prospectively (during implementation of the interventions) from the 23 facilities based on the steps shown above. Data were obtained from: key informant interviews, review of administrative data, FP utilization registers and observation of the time each provider spent with clients. The 
introduction of integrated services can be thought of as taking place in two phases: planning/preparation and service delivery.

The planning phase is used to prepare for the actual provision of services. Activities in this phase include: stakeholder meetings, site assessments, modifications to service delivery points, the production of IEC materials, and the training of service providers. The resources used to support these activities represent a one-time up-front investment and will depend upon the context in which the intervention takes place. As a result, one needs to be careful when generalizing these resource requirements to other settings.

The service delivery phase corresponds to the time when clients are receiving the integrated services. The additional resources required to provide integrated C\&T services were classified into three broad categories: labor, test kits, and other medical supplies. These resources are required on an on-going basis and therefore represent recurring costs to the program. The magnitude of these costs is sensitive to the volume of clients served but on a per client basis these costs can be inflated to estimate costs for different size programs. Assumptions made for service delivery cost estimation are outlined in the box below.

\begin{tabular}{|ll|}
$\begin{array}{l}\text { Cost } \\
\text { Category }\end{array}$ & Measurement and valuation of Cost Components \\
Labor & $\begin{array}{l}\text { Measurement: Additional time spent serving clients with integrated } \\
\text { services } \\
\text { Valuation: Government gross salary for 2006/2007, based on mean } \\
\text { grade J; working 22 days a month and } 8 \text { hours a day }\end{array}$ \\
$\begin{array}{l}\text { Quantities } \\
\text { and } \\
\text { combination } \\
\text { of rapid tests }\end{array}$ & $\begin{array}{l}\text { Measurement: Use of standard counseling and testing protocol. } \\
\text { Consumption of the Bioline Rapid HIV kits was estimated at 20\% of the } \\
\text { quantities of Determine Rapid HIV Kit used. Consumption of Unigold } \\
\text { (one of the rapid HIV test Kits) was estimated at four percent of the } \\
\text { Determine consumption (since consumption data on Unigold was often } \\
\text { incomplete or missing) }\end{array}$ \\
$\begin{array}{l}\text { Valuation: Unit costs of rapid test kits and reagents from KEMSA } \\
\text { Medical } \\
\text { supplies }\end{array}$ & $\begin{array}{l}\text { Measurement: Per protocol } \\
\text { Valuation: Unit costs of gloves obtained from KEMSA }\end{array}$ \\
\hline
\end{tabular}

When reporting on incremental costs, a distinction is made between financial and non-financial (opportunity) costs. While additional staff time is required to provide the integrated services, there are no additional labor expenditures required so this resource requires a re-allocation of existing resources. For the test kits and other medical supplies, there will need to be additional financial expenditures to source these supplies. The incremental financial and non-financial resources required are documented below. 
Planning / Preparation Costs: Prior to the intervention, FRONTIERS facilitated meetings with the $\mathrm{MOH}$ to reach consensus on the approaches to be used and the goals of integrating VCT and FP services. Job aids and client brochures were developed and produced and a total of 75 providers (47 in Thika district and 28 in Nyeri district) were trained. Each district held two training sessions so as not to disrupt service delivery in the pilot facilities. The cost per trainee per day was approximately $\$ 80$ for the testing model and $\$ 75$ for the referral model. Table A presents the estimated costs of planning and preparation activities which indicate the magnitude of up-front investments that may be required to replicate this process in other settings. Most of these costs were from the project and therefore outside of the $\mathrm{MOH}$.

\section{Table A: Planning and preparation costs by type of activity for both districts}

\begin{tabular}{|c|c|c|}
\hline \multicolumn{2}{|c|}{ Stakeholder Meetings } & $357,000(\$ 5,100)$ \\
\hline \multicolumn{2}{|c|}{ Development \& Production of Job Aids } & $469,560(\$ 6,708)$ \\
\hline \multicolumn{2}{|c|}{ Production of IEC Materials } & $336,000(\$ 4,800)$ \\
\hline \multicolumn{2}{|c|}{ Curriculum Development } & $1,447,600(20,6800)$ \\
\hline \multirow{3}{*}{$\begin{array}{l}\text { Training of } \\
\text { Providers }\end{array}$} & Testing Model : 28 providers, each trained for 9 days & $1,407,200(\$ 20,103)$ \\
\hline & Referral Model: 47 providers, each trained for 5 days & $1,228,444(\$ 17,549)$ \\
\hline & Sub-total & $2,635,644(\$ 37,652)$ \\
\hline \multicolumn{2}{|l|}{ TOTAL } & $4,888,804(\$ 69,840)$ \\
\hline
\end{tabular}

Incremental Costs of Service Delivery: The additional resources for provision of VCT within FP clinics for the testing model in Nyeri district are: labor, rapid HIV test kits and other medical supplies. Additional resources for the referral model in Thika district were labor, overhead and cost of buildings and equipment. The incremental cost per client served was computed for FP clients receiving the following services in each model: FP clients counseled and tested; FP clients who discussed the risk for HIV/STIs with provider; FP clients who were referred for testing and post-test counseling. The cost of labor was based on the time the health provider was observed spending in direct contact with the client. Using information obtained from the providers themselves and verified by the $\mathrm{MOH}$, the cost of labor per minute was derived as follows:

Cost $/$ minute $=$ Annual Salary $/($ Normal Work Days in a year, $22 \times 12$ months $) *$ Minutes per day

Table B shows the unit price of labor (per minute) and the difference in consultation time between baseline and end-line, to derive incremental labor cost per person ${ }^{7}$.

6 The total cost shown includes various costs such as transport refund to participants, full board costs for participants including trainers, incidental costs e.g. photocopying, out of pocket allowance, fuel refund, accommodation for drivers, purchase of training material e.g. stationery among others.

7 Similar calculations were applied in computing costs of reproductive health services in four CHAG hospitals (Boateng, et al, 2006). 
Table B: Unit price of labor and incremental labor time by district

\begin{tabular}{|l|c|c|c|c|c|}
\hline & Baseline survey & End line survey & \multicolumn{2}{|c|}{ Difference } \\
\hline Labor & $\begin{array}{c}\text { Unit price } \\
\text { (Kshs.) }\end{array}$ & $\begin{array}{c}\text { Average duration of } \\
\text { consultation time }\end{array}$ & $\begin{array}{c}\text { Average duration } \\
\text { of consultation } \\
\text { time }\end{array}$ & $\begin{array}{c}\text { Qty } \\
\text { used }\end{array}$ & $\begin{array}{c}\text { Incremental cost } \\
\text { per person } \\
\text { (Kshs) }\end{array}$ \\
\hline $\begin{array}{l}\text { Testing: } \\
\text { Nyeri }\end{array}$ & $\begin{array}{c}2.27 \text { per } \\
\text { minute }\end{array}$ & 12.40 & 15.43 & $\begin{array}{c}3.03 \\
\text { minutes }\end{array}$ & $2.27 \times 3.03=7$ \\
\hline $\begin{array}{l}\text { Referral: } \\
\text { Thika }\end{array}$ & $\begin{array}{c}2.27 \text { per } \\
\text { minute }\end{array}$ & 13.21 & 17.39 & $\begin{array}{c}4.18 \\
\text { minutes }\end{array}$ & $2.27 \times 4.18=9$ \\
\hline
\end{tabular}

The incremental time for Thika District was higher (4.18 minutes) than that of Nyeri District (3.03 minutes), although the reasons for the differences were not investigated. However, background data shows that facilities in Thika are better staffed than those in Nyeri. For instance, facilities in Thika have an average of 7 enrolled nurses while those in Nyeri have 4 enrolled nurses. Findings from the focus group discussions seemed to confirm that providers especially in Thika had devised coping mechanisms for dealing with workload despite the staff shortage such as reporting to work early, using lunchtime break and any other free time to attend to the clients, and using other staff members such as Public Health Technicians to educate clients on FP and VCT. These actions seemed to increase the time available for provider-client consultations. On the other hand, given that Nyeri was worse off with regard to staff shortage when compared to Thika, it is probable that consultations took a shorter time in order to serve all the clients in the testing model without turning any of them away. The fact that providers in Nyeri did not mention employing any coping mechanisms seemed to suggest that they did not gain additional contact time with their clients.

Cost of rapid test kits and supplies: The cost of rapid HIV test kits and gloves used in performing an HIV test are presented in the Table C. In Nyeri a total of 4,748 HIV test kits were used and 4000 pairs of gloves during the intervention. The total cost was Kenya Shillings 355,676 (approximately $\$ 5,472$ )

Table C: Cost of HIV rapid test kits and disposal gloves (Nyeri district)

\begin{tabular}{|l|c|c|c|c|c|}
\hline Item & $\begin{array}{c}\text { Qty of items } \\
\text { per pack }\end{array}$ & $\begin{array}{c}\text { Price per } \\
\text { pack }\end{array}$ & $\begin{array}{c}\text { Unit price } \\
\text { (Kshs.) }\end{array}$ & $\begin{array}{c}\text { No. of tests/items } \\
\text { used }\end{array}$ & Total cost \\
\hline Determine & 100 & $6,925.10$ & 69.25 & 3829 & 265,172 \\
\hline Bioline & 20 & $1,358.00$ & 67.90 & 766 & 52,001 \\
\hline Unigold & 20 & $2,520.00$ & 126.00 & 153 & 19,303 \\
\hline $\begin{array}{l}\text { Disposable } \\
\text { gloves }\end{array}$ & 50 & 240.00 & 4.80 & 4000 & 19,200 \\
\hline Total Cost & \multicolumn{5}{|l}{} \\
\hline
\end{tabular}

Source of the price for supplies: Kenya Medical Supplies Agency Procurement Department

Cost of additional supervisory visits: In order to reinforce the new methods of counseling clients, the Population Council provided additional supervisory visits to complement the ongoing supervision visits provided by the $\mathrm{MOH}$. The resources used for these activities totaled 400,050 Kshs or $\$ 5,715$ (Table D). The table below indicates how these costs varied by model and type of facility. The variation is due to differences in travel distances, number of facilities, and number of visits per facility. 
Table D: Cost of additional supervision during intervention by model and level of care

\begin{tabular}{|l|c|c|}
\hline Model & Level of Care & Supervision Costs (Kshs) \\
\hline \multirow{4}{*}{ Testing } & Hospital $(n=1)$ & 45,929 \\
\cline { 2 - 3 } & Health Centre $(n=4)$ & 74,834 \\
\cline { 2 - 3 } & Dispensary $(n=4)$ & 35,687 \\
\cline { 2 - 3 } & Sub-Total $(n=9)$ & 156,450 \\
\hline \multirow{4}{*}{ Referral } & & \\
\cline { 2 - 3 } & Hospital $(n=2)$ & 93,610 \\
\cline { 2 - 3 } & Health Centre $(n=8)$ & 117,048 \\
\cline { 2 - 3 } & Dispensary $(n=4)$ & 32,942 \\
\cline { 2 - 3 } & Sub-Total $(n=14)$ & 243,600 \\
\hline
\end{tabular}

Total Incremental Cost for Service Delivery: The annual labor costs were higher in Thika compared to Nyeri District, which can be explained by the fact that more clients $(36,206)$ were seen in Thika's 14 health facilities when compared to those who were seen in 9 health facilities in Nyeri $(23,277)$. A summary of total costs by resource categories and average cost by model are shown in Table E.

Table E: Incremental costs by resource categories and average incremental cost by model (Kshs)

\begin{tabular}{|l|c|c|c|c|}
\hline & \multicolumn{2}{|c|}{$\begin{array}{c}\text { Incremental cost by } \\
\text { resource category }\end{array}$} & \multicolumn{2}{c|}{$\begin{array}{c}\text { Average incremental cost for each FP } \\
\text { client per resource area based on FP } \\
\text { workload }\end{array}$} \\
\hline Resource category & Nyeri & Thika & Testing (Nyeri) & Referral (Thika) \\
\hline Labor & 160,102 & 343,544 & 6.90 & 9.50 \\
\hline Test Kits & 336,476 & & 14.50 & - \\
\hline Gloves & 19,200 & & 0.80 & - \\
\hline Additional Supervision & 156,450 & 243,600 & 6.7 & 7 \\
\hline Total & 672,228 & 587,144 & 28.90 & 16.50 \\
& $(\$ 9,603)$ & $(\$ 8,388)$ & $(\$ 0.41)$ & $(\$ 0.24)$ \\
\hline
\end{tabular}

The average additional contact time was 3.03 minutes in Nyeri health facilities compared to 4.18 minutes in the Thika health facilities. The additional annual labor cost (total contact time $\mathrm{x}$ cost per minute) amounted to Kshs 70,529 (\$1,085) and Kshs 151341 (\$2,328) in Nyeri and Thika respectively. For both models, the average cost by level of care was low for dispensaries when compared to hospitals (Table F). The differences in hospital and dispensary costs were more prominent in the Thika model where hospital costs were almost three times more. However this is related to client load and number of staff available.

Out of the 23,277 clients who attended for FP services in the pilot facilities in Nyeri District, 3,829 were tested for HIV. The total amount of service delivery costs (financial resources) spent in the testing model was Kshs. 672,228 including the opportunity cost for labor. Incremental average cost per FP client tested for HIV was Kshs.176 (\$2.5). 
Table F: Average incremental service delivery costs by type of resource and level of care in the two models

\begin{tabular}{|c|c|c|c|c|c|c|}
\hline Level of Care & $\begin{array}{l}\text { Labor } \\
\text { (Kshs) }\end{array}$ & $\begin{array}{l}\text { Test } \\
\text { Kits } \\
\text { (Kshs) }\end{array}$ & $\begin{array}{l}\text { Gloves } \\
\text { (Kshs) }\end{array}$ & $\begin{array}{l}\text { Additional } \\
\text { Supervision } \\
\text { (Kshs) }\end{array}$ & $\begin{array}{l}\text { Total } \\
\text { (Kshs) }\end{array}$ & $\begin{array}{l}\text { Average cost/per } \\
\text { facility type (Kshs) }\end{array}$ \\
\hline \multicolumn{7}{|l|}{$\begin{array}{l}\text { Nyeri District } \\
\text { (Testing model) }\end{array}$} \\
\hline Hospital-Nyeri PGH & 46,430 & 97,578 & 5,339 & 45,929 & 195,276 & $195,276(\$ 2,790)$ \\
\hline Health centers & 76,849 & 161,509 & 10,45 & 74,834 & 323,648 & $80,912(\$ 1,156)$ \\
\hline Dispensaries $(n=4)$ & 36,823 & 77,389 & 3,405 & 35,687 & 153,304 & $38,326(\$ 548)$ \\
\hline Sub Total $(n=9)$ & 160,10 & 336,476 & 19,20 & 156,450 & 672,228 & $74,692(\$ 1,067)$ \\
\hline $\begin{array}{l}\text { Thika District } \\
\text { (Referral model) }\end{array}$ & Labor & $\begin{array}{l}\text { Test } \\
\text { Kits }\end{array}$ & Gloves & $\begin{array}{l}\text { Additional } \\
\text { Supervision }\end{array}$ & Total & $\begin{array}{l}\text { Average cost/per } \\
\text { facility type }\end{array}$ \\
\hline Hospital $(n=2)$ & 132,02 & 0 & 0 & 93,610 & 225,633 & $112,816(\$ 1,612)$ \\
\hline Health centers & 165,07 & 0 & 0 & 117,048 & 282,119 & $35,265(\$ 504)$ \\
\hline Dispensaries $(n=4)$ & 46,450 & 0 & 0 & 32,942 & 79,392 & $19,848(\$ 284)$ \\
\hline Sub Total $(n=14)$ & 343,54 & 0 & 0 & 243,600 & 587,144 & $41,939(\$ 599)$ \\
\hline
\end{tabular}




\section{FOR MORE INFORMATION, CONTACT:}

Frontiers in Reproductive Health

Population Council

4301 Connecticut Avenue, N.W.

Suite 280

Washington, D.C. 20008

USA

Telephone: $\quad$ 202-237-9400

Facsimile: 202-237-8410

E-mail: frontiers@pcdc.org

Website: $\quad$ www.popcouncil.org

AfricA

Population Council Regional Office

P.O. Box 17643

Nairobi

Kenya

Telephone: $\quad$ 254-2-2713480/1/2/3

Facsimile: $\quad$ 254-2-2713479

E-mail: $\quad$ publications@pcnairobi.org

Asia AND the Near EAst

Population Council Regional Office

Ground Floor, Zone 5A

India Habitat Center

Lodi Road

New Delhi 110003

India

Telephone: 91-11-461-0913

Facsimile: $\quad 91-11-464 \backslash 2903$

E-mail: $\quad$ frontiers@pcindia.org

Latin America AND the CARIBbeAn

Population Council Regional Office

Escondida 110

Villa Coyoacan

04000 Mexico, D.F.

Mexico

Telephone:

52-5-659-8537

Facsimile:

52-5-554-1226

E-mail:

disemina@popcouncil.org.mx

\section{Population Council}

The Population Council is an international, nonprofit, nongovernmental institution that seeks to improve the well-being and reproductive health of current and future generations around the world and to help achieve a humane, equitable, and sustainable balance between people and resources. The Council conducts biomedical, social science, and public health research and helps build research capacities in developing countries. Established in 1952, the Council is governed by an international board of trustees. Its New York headquarters supports global network of regional and country offices.

\section{FRONTIERS \\ IN REPRODUCTIVE HEALTH}

FRONTIERS is funded by the Office of Population of the UNITED STATES AGENCY FOR INTERNATIONAL DEVELOPMENT (USAID) under the terms of Cooperative Agreement Number HRN-A-00-98-00012-00 